\title{
A Near-Infrared Stellar Census of Blue Compact Dwarf Galaxies: The Wolf-Rayet Galaxy I Zw $36^{1}$
}

\author{
Regina E. Schulte-Ladbeck \\ University of Pittsburgh, Pittsburgh, PA 15260, USA \\ rsl@phyast.pitt.edu \\ Ulrich Hopp \\ Universitätssternwarte München, München, FRG \\ hopp@usm. uni-muenchen.de \\ Laura Greggio \\ Osservatorio Astronomico di Bologna, Bologna, Italy, and Universitätssternwarte München, \\ München, FRG \\ greggio@usm.uni-muenchen.de \\ Mary M. Crone \\ Skidmore College, Saratoga Springs, NY 12866, USA \\ mcrone@skidmore.edu \\ Igor O. Drozdovsky \\ University of Pittsburgh, Pittsburgh, PA 15260, USA, and University of St. Petersburg, \\ St. Petersburg, Russia
}

\footnotetext{
${ }^{1}$ Based on observations made with the NASA/ESA Hubble Space Telescope obtained from the Space Telescope Science Institute, which is operated by the Association of Universities for Research in Astronomy, Inc., under NASA contract NAS 5-26555.
} 
dio@phyast.pitt.edu

Received __; accepted _ 


\begin{abstract}
We report the results of near-IR imaging in $\mathrm{J}$ and $\mathrm{H}$, of $\mathrm{I} \mathrm{Zw} 36\left(\approx \mathrm{Z}_{\odot} / 14\right)$ with the Hubble Space Telescope. Whereas imaging with the pre-COSTAR Faint Object Camera (FOC) previously resolved hot and massive stars in the near-UV, the NICMOS data furnish a census of the cool, intermediate- and low-mass stars. There clearly was star formation in I Zw 36 prior to the activity which earned it its Blue Compact Dwarf/Wolf-Rayet galaxy classification. The detection of luminous, asymptotic giant branch stars requires that stars formed vigorously several hundred Myr ago. The well-populated red giant branch indicates stars with ages of at least 1-2 Gyr (and possibly older than $10 \mathrm{Gyr}$ ). We use the tip-ofthe-red-giant-branch method to derive a distance of $\geq 5.8 \mathrm{Mpc}$. This is the third in a series of papers on near-IR - resolved Blue Compact Dwarf galaxies. We notice that the color-magnitude diagrams of VII Zw 403, Mrk 178, and I Zw 36 do not exhibit the gaps expected from an episodic mode of star formation. Using simulated color-magnitude diagrams we demonstrate for I Zw 36 that star formation did not stop for more than a few $10^{8}$ yrs over the past $10^{9} \mathrm{yrs}$, and discuss the implications of this result.

Subject headings: Galaxies: compact — galaxies: dwarf — galaxies: Wolf-Rayet — galaxies: evolution — galaxies: individual (I Zw $36=$ UGCA $281=$ Mrk 209 = Haro 29) — galaxies: stellar content
\end{abstract}

\title{
1. Introduction
}

Nearly all galaxies in the low-redshift Universe show clear signs of maturity: colors and spectra which suggest an older population of stars in addition to any recent star formation. 
There are certain nearby galaxies, however, which have long been suspected of youth. Extreme Blue Compact Dwarfs (BCD) like I Zw 18 exhibit extremely low metal abundances, very blue colors, and large H-I reservoirs, and could be genuine proto-galaxies experiencing their first star-forming event at the present epoch (Sargent \& Searle 1970; Searle \& Sargent 1972; Searle, Sargent \& Bagnuolo 1973; Izotov \& Thuan 1999). It has been argued that if BCDs instead are old galaxies, then they can nevertheless not have experienced more than a few, short starbursts separated by long quiescent periods. Chemical evolution models predict that interstellar abundances rise above observational values after only a few star-forming episodes (e.g., Kunth \& Sargent 1986; Marconi, Matteucci \& Tosi 1994). With star formation rates (SFR) between 0.01 and $10 \mathrm{M}_{\odot} \mathrm{yr}^{-1}$ and typical H-I masses of a few times $10^{8} \mathrm{M}_{\odot}$, the gas depletion time scales for BCDs range from between a few times $10^{7}$ to $10^{10}$ years (Thuan 1991). Infrequent, short bursts have thus become widely accepted as the paradigm for the star formation mode of BCDs (e.g. Thuan et al. 1999). This interpretation only recently has come under re-consideration (Legrand et al. 2000).

In the 1980s, deep ground-based CCD imaging revealed that most (>90\%) of BCDs harbor an elliptical, red, low surface brightness sheet of background stars (Loose \& Thuan 1986; Kunth, Maurogordato \& Vigroux 1988; also Papaderos et al. 1996; Telles, Melnick \& Terlevich 1997; Doublier et al. 2000). With the advent of HST, it has become possible to resolve the background sheets of the most nearby BCDs into red giant branch (RGB) and asymptotic giant branch (AGB) stars. This avoids the degeneracy of spatially integrated broad-band photometry, which cannot distinguish intermediateor low-mass stars from the massive, red supergiants (RSG). This paper on I Zw 36 $\left(12+\log (\mathrm{O} / \mathrm{H})=7.77\right.$, Izotov \& Thuan $\left.1999 ; \approx \mathrm{Z}_{\odot} / 14\right)$ is the third in a series of papers which address the near-IR photometry of resolved stars in BCDs. The color-magnitude diagrams (CMDs) of VII Zw $403\left(12+\log (\mathrm{O} / \mathrm{H})=7.69\right.$, Izotov \& Thuan 1999; $\left.\approx \mathrm{Z}_{\odot} / 17\right)$ and Mrk $178\left(12+\log (\mathrm{O} / \mathrm{H})=7.95 ;\right.$ Kobulnicky \& Skillman 1996; $\left.\approx \mathrm{Z}_{\odot} / 10\right)$ were discussed in 
previous papers. Both optical and near-IR CMDs were used to derive the star formation history of VII Zw 403 (Schulte-Ladbeck, Crone \& Hopp 1998, hereafter SCH98; Lynds et al. 1998; Schulte-Ladbeck et al. 1999, hereafter SHCG99; Schulte-Ladbeck et al. 1999, hereafter SHGC99), while near-IR CMDs formed the basis of the stellar census of Mrk 178 (Schulte-Ladbeck et al. 2000, hereafter SHGC00). In both galaxies, star formation was detected at times that pre-date the current activity, and both are at least 1-2 Gyr old. The very nearby, newly identified BCD NGC 6789 was resolved from the ground (Drozdovsky \& Tikhonov 2000); and we just detected the red giant branch with HST's WFPC2 (Drozdovsky et al. 2001a). Meanwhile, we also resolved red giants with WFPC2 in the BCD UGCA 290, at a distance of about $6.7 \mathrm{Mpc}$ on the line of sight to the Canes Venatici cloud (Crone et al. 2000). We note that this BCD does not share the dominant, "iE" morphology; rather, it is a case of a background sheet that was largely hidden to ground-based photometry underneath the star-forming regions. This adds another two BCDs for which an old stellar component has been directly detected; and the results for UGCA 290 suggest that "Baade's red sheet" occurs in an even larger fraction of BCDs (over $90 \%$ ) than was previously assumed.

I Zw $36=$ UGCA $281=$ Mrk $209=$ Haro 29 is an excellent candidate with which to test existing formation scenarios for BCDs. It is nearby, has a low oxygen abundance and large H-I content, and has been proposed to be a galaxy currently undergoing its very first starburst (Fanelli, O'Connell \& Thuan 1988). Its extinction-corrected, total apparent B magnitude from the RC3 is 14.94, which, using our new distance modulus of 28.8, translates into an absolute magnitude of -13.9 .

A comprehensive, multifrequency study of I Zw 36 was presented by Viallefond \& Thuan (1983). They found a core-halo structure in H-I gas, with the visible star-forming regions situated near that core, but slightly shifted with respect to the peak in H-I 
column density. This appears to be quite a common occurrence in BCDs. Van Zee, Skillman \& Salzer (1998) observed five BCDs in H-I and noticed they exhibit strong central concentrations in their neutral gas distributions, but with moderate depressions in column density over the most intensely star-forming regions. The H-I data of I Zw 36 of Viallefond \& Thuan (1983) also yielded the distance which has henceforth been used in the literature; with a Hubble constant of $75 \mathrm{~km} \mathrm{~s}^{-1} \mathrm{Mpc}^{-1}$, they determined 4.6 Mpc. The H-I mass is then about $4 \times 10^{7} \mathrm{M}_{\odot}$ (at a distance of $5.8 \mathrm{Mpc}$, the H-I flux listed in Table 1 of Viallefond \& Thuan corresponds to an H-I mass of $6 \times 10^{7} \mathrm{M}_{\odot}$ ), and the virial mass about $5-7$ times the H-I mass. The latter result has pointed to the potential importance of dark matter in this BCD. The nebular lines in the optical spectrum and the blue-UV continuum show that star formation has been active in recent times. Interestingly, I Zw 36 has an interstellar CO detection despite of its rather low O abundance (Tacconi \& Young 1987). Near-IR photometry was interpreted by Viallefond \& Thuan to indicate the presence of giant stars, rather than a contribution to the integrated colors by RSGs from the present star formation event. Using stellar CO indices to identify RSGs in two BCDs, this interpretation of near-IR colors was later called into question by Campbell \& Terlevich (1984).

In their CCD survey of BCDs, Loose \& Thuan (1986) identified an extended, elliptical background sheet underlying the compact, active star formation core of I Zw 36 (see their Fig 1). They classify I Zw 36 as an "iE" BCD; this is the most common morphological type and considered characteristic of the BCD phenomenon.

I Zw 36 was included in the IUE UV spectral synthesis study of BCD stellar populations by Fanelli, O'Connell \& Thuan (1988), who found that its spectral energy distribution was accounted for by young, hot, O and B stars, with no light from stars cooler than about B6. Fanelli, O'Connell \& Thuan pointed to the unique nature of I Zw 36: unlike any other BCD in their sample, it could be undergoing its first starburst at the present epoch. 
This finding prompted a follow-up UV imaging study with the pre-Costar HST (Deharveng et al. 1994). Using the FOC with a variety of UV and blue filters, Deharveng et al. successfully resolved massive stars in the inner 11 square $\operatorname{arcsec}$ of I Zw 36 . They noticed that the integrated F175W-F430W color of the galaxy is redder than expected from the current star formation. Ruling out contamination by RSGs, as no RSGs appeared on their CMDs, they suggested the presence of an older, underlying stellar population in addition to the young and massive stars.

Papaderos et al. (1996) subsequently obtained deep, ground-based optical imaging of I Zw 36. They drew attention to a second star-forming region spatially separated from the prominent one, and proposed that a previous episode of star formation took place here. In addition, they found that the galaxy becomes redder with increasing distance from the core. They suggested this underlying low-surface-brightness component represents an older stellar population formed prior to the present starburst.

Most recently, the brightest supergiants of I Zw 36 were resolved in the optical with ground-based imaging. Makarova, Karachentsev \& Georgiev (1997) derive a distance of 5.7 Mpc for I Zw 36; placing this galaxy more than $1 \mathrm{Mpc}$ further out than was previously assumed. I Zw 36 lies on the line of sight to the Canes Venatici cloud of galaxies.

In this paper, we show that I Zw 36 resolves into single stars in the near-IR with HST. We find that our near-IR CMDs clearly reveal the presence of RSGs in this galaxy. Therefore, where young and old stellar populations spatially overlap, integrated colors cannot distinguish the nature of the stellar content (see also SHGC99). The near-IR CMDs exhibit resolved AGB and RGB stars, the descendents of intermediate- and low-mass stars. We derive a minimum distance of approximately $5.8 \mathrm{Mpc}$, and a minimum age of 1-2 Gyr for this galaxy. 


\section{Observations and reductions}

We observed I Zw 36 on 1998 July 7 as part of our GO program 7859. The NIC2 camera, which has a field of view about 19 arcsec across, was centered at (J2000) R.A. 12:26:16 and Dec. 48:29:39. Figure 1 shows the location of the NIC2 observation relative to the Digitized Sky Survey (DSS) image of the galaxy. The NIC2 field overlaps with the FOC field. (The NIC2 total field shown in Fig. 2 is slightly larger than the single NIC2 field superposed in Fig. 1, because the camera was dithered from one exposure to the next.) We shall not discuss the data in the NIC1 field, whose location is also indicated on Fig. 1. The NIC1 field contains very few (less than ten) stars, primarily because the sensitivity of this camera is lower than that of NIC2, and of course also because of its smaller size and location near the edge of the visible galaxy body. While future data mining might allow us to gain information on any potential high- and intermediate-mass stars at this location within I Zw 36, our present study focuses only on the more interesting, NIC2 results.

The data were obtained in the F110W and the F160W filters, which are similar to the ground-based J and $\mathrm{H}$ bands. The data acquisition consisted of two sets of three exposures in F110W, and one set of three in F160W. The first set of F110W exposures used integration times of about $896 \mathrm{~s}$ each. All other exposures were all about $960 \mathrm{~s}$ in length. The telescope was dithered by $1^{\prime \prime}$ between exposures. The data were read out in MULTIACCUM mode.

The data were re-reduced following the steps described in SHGC99, and again in SHGC00. In brief, the exposures were cleaned of cosmic rays; a temperature-dependent dark was applied; they were inspected for cosmic-ray persistence; and the pedestal was removed. All exposures were used in the combined, final images in F110W and F160W. The total integration times were thus about $5568 \mathrm{~s}$ in F110W, and $2880 \mathrm{~s}$ in F160W.

Fig. 2a,b illustrates how well I Zw 36 resolves into single stars with NIC2. Comparing Fig. 2 with Fig. 1 of Deharveng et al. (1994), we recognize the most prominent H-II regions 
were captured by both the near-UV and the near-IR imagers.

Single-star photometry was conducted using DAOPHOT (Stetson, Davis, \& Crabtree 1990). The conversion from instrumental magnitudes to absolute photometry followed the NICMOS Photometric Calibration CookBook, with the photometric zero-points in the Vegamag system as provided by the NICMOS team. This yielded two photometry lists, F110W and F160W, in the HST Vegamag system. Figure 3 shows the error distributions for the photometry. The photometry reaches limiting magnitudes of about 26.5 in F110W, and 25.5 in F160W. We merged the two star lists requiring a positional source coincidence of better than 1.5 pixels or $\approx 0^{\prime}{ }^{\prime} 1$. There are 712 objects with coincident positions in F110W and F160W.

Completeness tests were conducted using the DAOPHOT/ADDSTAR routine, by adding 100 false stars in each image and for each magnitude interval in the data. For each magnitude bin, 10 such tests were carried out. An artificial star was considered to be recovered if the difference between input and output magnitude was less than 0.75 . The percentage of recovered stars is shown in Fig. 4. These tests indicate that the data are better than $80 \%$ complete to a magnitude of about 24 in F110W and about 23.5 in F160W.

Figure 5 shows CMDs constructed from these data. Subsequent CMDs in the ground-system (J, H) use the transformations which we derived in SHGC99, based on the NICMOS team's information on standard-star observations from their photometry WWW site. Figure 6 shows the CMDs transformed to ground-based J and H, on the same scale as those of Fig. 5 for comparison.

Another group has recently published transformations from F110W, F160W, to ground based J and H in the CIT/CTIO system (Stephens et al. 2000). This group draws on observations of red, solar-metallicity stars in Baade's window. We compared their transformation to our transformation, and found there was no significant difference which 
would affect our results. For example, a "typical" stellar color near the tip of the red giant branch is F110W-F160W=1.0 in the HST Vegamag system. In our ground system, this corresponds to a J-H color of $0.79 \pm 0.15$. In the ground system of Stephens et al., it corresponds to $0.61 \pm 0.14$. This amounts to a systematic color difference of $0.18 \pm 0.21$ between the two systems. We will continue to use our own transformations. Our distance calibration, and the transformation of stellar-evolution tracks used in the simulators, are based on them (see SHGC99). For the above color, the difference in $\mathrm{H}$ magnitudes introduced by the different calibrations is $0.05 \pm 0.16$. Thus the distance determination is not affected within the errors by our choice of calibration.

We note that any tracks which we overplot on subsequent CMDs originate from tracks in the flight system, which were transformed using the same transformation as the data whenever comparisons in the ground system are carried out. Thus, our data and our tracks are on a consistent system.

\section{Results}

Because the field of the NIC2 camera is so small and because I Zw 36 is situated at high galactic latitude $\left(68^{\circ}\right)$, we expect the contamination of the CMDs by galactic foreground stars to be very small. Based on the data of Ratnatunga \& Bahcall (1985), we determine a galactic foreground of 0.7 stars in the NIC2 field of view (down to a limiting magnitude of $\mathrm{V}=26$ ). Thus, even if this estimate should for some reason be by as much as a factor of 10 too small, it is still straightforward to interpret the CMDs. The CMDs contain over 700 stars that must belong almost exclusively to I Zw 36 .

The foreground galactic extinction towards I Zw 36 is negligible: $\mathrm{A}_{B}=0.000$ from H-I maps (Burstein \& Heiles 1984); $\mathrm{A}_{B}=0.065$, which corresponds to $\mathrm{A}_{J}=0.013$ and $\mathrm{A}_{H}=0.009$ 
from IRAS maps (Schlegel, Finkbeiner \& Davis 1998, and see NED).

Like that of many other star-forming dwarf galaxies (e.g. Mas-Hesse \& Kunth 1999), the internal extinction of I Zw 36 comes out to be different from spectroscopy and imaging. Deharveng et al. (1994) comment on how their CMDs do not suggest any substantial extinction in the UV, whereas the spectroscopic Balmer decrement used by Viallefond \& Thuan (1983) indicates $\mathrm{E}(\mathrm{B}-\mathrm{V})=0.3$. They also note that the UV spectrum of Fanelli et al. (1988) indicates a very low internal extinction. We assume that, as in other star-forming dwarfs, reddening is much larger for the ionized gas than for the diffuse stellar population (Calzetti et al. 1997) and that the internal extinction is negligibly small in the near-IR CMDs.

The CMDs of Figs. 5 and 6 are characterized by a strong red plume, and a comparatively weak blue plume (spread out around $\mathrm{J}-\mathrm{H}=0$ ). We definitely detect stars that qualify as RSGs belonging to I Zw 36. The near-IR observations thus complement the near-UV-optical observations from which the presence of RSGs remained doubtful (Deharveng et al. 1994). Most of the stars in the near-IR CMDs appear at faint magnitudes at the bottom of the red plume. This is the red tangle which contains the RGB. Depending on the specific star formation history, some combination of AGB stars and even core-He-burning stars can contribute to this feature. The dashed line of Fig. 5 marks the magnitude at which we identify the TRGB (see below). Above the TRGB, we expect to see AGB and RSG stars. There is indeed a considerable number of red stars above the RGB. The blue plume can include main-sequence (MS), blue-loop (BL), and blue-supergiant (BSG) stars. It is not well defined owing to the large measurement errors for blue stars in the data. 


\subsection{The distance}

We use the tip-of-the-red-giant-branch (TRGB) method to constrain the distance to I Zw 36. There are advantages and disadvantages to attempting the TRGB method in the near-IR. Of advantage is the fact that RGB stars are brighter in $\mathrm{J}$ and $\mathrm{H}$ than in $\mathrm{I}$ and $\mathrm{V}$, the two colors conventionally used in the TRGB method (Lee, Freedman \& Madore 1993). We constructed a calibration for the TRGB method in J and H using VII Zw 403 HST data, stellar-evolution models, and globular cluster data (SHGC99) ${ }^{2}$. Of disadvantage is the fact that CMDs of star-forming galaxies, and those that involve near-IR colors in particular, always show a large number of AGB stars. Our near-IR observations of VII Zw 403 and Mrk 178 revealed that AGB stars are a strong stellar component (SHGC00); the same is true for I Zw 36. Low- as well as intermediate-mass AGB stars can overlap in color and luminosity with RGB stars near the TRGB. This causes luminosity functions of stars along the red plume to exhibit more of a gradual variation rather than the desired step function that would furnish a well-defined, TRGB magnitude for the distance determination. Finally, of all our near-IR observations, the errors and completeness fractions are poorest for I Zw 36. This by itself suggests I Zw 36 is the most distant of the three galaxies.

In determining the distance to I Zw 36, we start by inspecting the luminosity functions. The luminosity functions of 356 stars selected to have colors in the range $0.7<(\mathrm{F} 110 \mathrm{~W}-\mathrm{F} 160 \mathrm{~W})<1.4$ and binned in $0.1 \mathrm{mag}$ intervals are shown in Fig. 7. We applied an edge-detection, Sobel filter (cf. Sakai, Madore \& Freedman 1996). The central value of the luminosity functions was varied in steps of 0.05 mag to reduce the dependence of the results upon the particular choice of bin center. The luminosity functions were then smoothed using a sliding average of three points, and a Sobel kernel, $[-1,0,+1]$, was applied.

\footnotetext{
${ }^{2}$ Drozdovsky et al. (2001b) confirm this calibration using a larger number of lowmetallicity galaxies.
} 
The position of the TRGB is indicated by the highest peak that falls shortward of the completeness limit, at $\mathrm{m}_{F 110 W_{0}, T R G B}=24.52$ and $\mathrm{m}_{F 160 W_{0}, T R G B}=23.46$, respectively. Because we adopted a $0.1 \mathrm{mag}$ binning in the initial luminosity functions, the formal error on these positions is \pm 0.05 mag.

We derive the distance modulus of I Zw 36 using the calibration established for VII Zw 403 in SHGC99: $\mathrm{M}_{F 110 W_{0}, T R G B}=-4.28 \pm 0.10 \pm 0.18$ and $\mathrm{M}_{F 160 W_{0}, T R G B}=$ $-5.43 \pm 0.10 \pm 0.18$, where the first error is the statistical error and is dominated by how well we can determine the location of the TRGB in VII Zw 403, and the second is the systematic error primarily due to the RR Lyrae distance calibration of the TRGB (see SCH98). Application of the above calibration assumes that the RGB stars of I Zw 36 have the same metallicity as those in VII Zw $403([\mathrm{Fe} / \mathrm{H}]=-1.92)$. The F110W-F160W color at the TRGB of I Zw 36 is certainly consistent with assuming a low metallicity for the RGB. In what follows, the errors quoted are those that refer only to our statistical errors. Applying this calibration directly yields the following distance moduli for I Zw 36 using the TRGB in F110W and F160W, respectively: $28.8( \pm 0.1)$ and $28.9( \pm 0.1)$, corresponding to distances of 5.8 $( \pm 0.3) \mathrm{Mpc}$ and $6.0( \pm 0.3) \mathrm{Mpc}$.

In SHGC99, we derived a more general calibration over a wider range of metallicities by comparing the H-band magnitude of the TRGB of VII Zw 403 with that of GCs and stellar evolution models. The TRGB in $\mathrm{H}$ is constant as a function of metallicity for a wide range of low metallicities: $\mathrm{M}_{H_{0}, T R G B}=-5.5 \pm 0.1$ for $-2.3<[\mathrm{Fe} / \mathrm{H}]<-1.5$. The transformed $\mathrm{H}$ magnitude at the TRGB is $\mathrm{m}_{H_{0}, T R G B}=23.4 \pm 0.1$ (where the error is our transformation uncertainty). This suggests an H-band based distance modulus of $28.9( \pm 0.2)$, corresponding to a distance of $5.9( \pm 0.4) \mathrm{Mpc}$.

To summarize, the distances derived using F110W, F160W and H agree very well. We shall therefore assume a distance modulus of 28.8 for our minimum distance to I Zw 36 . 
We inspected Fig. 5 to double-check that the inferred "jumps" of stellar numbers in the luminosity functions, on which we base the distance estimate, coincide with where we would place the TRGB on the CMDs. As was the case in Mrk 178 (see SHGC00), the top of the red tangle which includes the RGB is quite wide, and it is markedly tilted in the [(F110-F160), F160] CMD (see Fig 5). We could place the TRGB in F160W at 0.2 mag fainter, and it would still lie within the rather broad rise of the F160W luminosity function shown in Fig. 7. We also notice that we find the TRGB at magnitudes where the completeness of the data has already begun to drop to around $80 \%$ (see Fig. 4 and section 2). Our artificial star experiments show that the recovered stars are systematically brighter than the input stars at these magnitudes. This is due to blending, which has the effect of smearing out the discontinuity of the number counts at the TRGB, artificially brightening its location. Therefore, the real uncertainty of the tip detection could be larger than the formal error we assumed so far. While it is unlikely that we find the TRGB at a location that is too faint, the above considerations indicate it is probable that we find it too bright. Therefore, a more realistic error on the distance modulus is not symmetrical. We adopt $28.8_{-0.1}^{+0.2}$ as our best estimate. As stated above, the systematic errors amount to an additional uncertainty of \pm 0.18 . The distance of $5.8 \mathrm{Mpc}$ used throughout this paper represents the minimum distance to I Zw 36.

As we do not have a metallicity calibration for the RGB in the near-IR, our method gives the smallest distance which is consistent with the data. If, however, the RGB population of I Zw 36 is more metal-rich than assumed above, then the TRGB indicates a larger distance modulus. This is due to the metallicity dependence of $\mathrm{M}_{H}$ (as illustrated in Fig. 11 of SHGC99). The high-metallicity distance modulus of I Zw 36 (corresponding to the TRGB on the $\mathrm{Z}=0.004$ tracks) is 29.5 ; this is our maximum distance. Therefore, an additional uncertainty of +0.7 in the distance modulus comes about due to unknown RGB metallicity. 
Our minimum distance agrees very well with the distance determined from the brightest stars method, 5.7 Mpc, by Makarova, Karachentsev \& Georgiev (1997). In the subsequent sections of this paper, we compare the I Zw 36 data with theoretical tracks of stellar evolution and with simulated CMDs. We point out that any "high-metallicity" assumption for the RGB corresponds to a "long" distance. From our previous, optical work on VII Zw 403 and UGCA 290 we have a slight preference for the "low-metallicity" assumption. The assumption of a low-metallicity RGB is supported by the empirical fact that other dwarf galaxies with low oxygen abundances in their H-II regions also tend to have low iron abundances of their old stellar populations (cf. Fig. 1 of Kunth \& Östlin 2000). However, without better knowledge of the metallicity of the RGB stars in I Zw 36, we emphasize that the "low-metallicity" or "short" distance used here must be regarded as a lower limit to the true distance of I Zw 36.

\subsection{The stellar content}

Which of the features observed on the CMDs should be considered real, and which artifacts of the errors? In Figure 8, we display [(J-H $\left.)_{0}, \mathrm{H}_{0}\right]$ CMDs of I Zw 36 with three values of the DAOPHOT errors applied as a selection criterion. These may be compared with Fig. 6. When both the $\mathrm{J}$ and $\mathrm{H}$ errors are smaller than 0.1 mag, the dominant contribution is a bright, red feature attributable to RSGs and luminous AGB stars. We see only very few BSGs and/or massive MS stars. When the J and H errors are smaller than 0.15 mag, both the blue and red plumes of the CMD become better populated. We

also pick up some RGB stars; however, the TRGB is not yet obvious (although there is a "break" in the data of the red plume below $\mathrm{H}_{0}$ of about $\left.23 \mathrm{mag}\right)$. If the magnitude errors are further increased to $0.2 \mathrm{mag}$, all the salient features of the CMD are apparent. At this error cut, the thermally-pulsing, or TP-AGB stars, defined as having $(\mathrm{J}-\mathrm{H})_{0}$ colors redder 
than 1 mag (beyond the end of our tracks, see Fig. 9), become visible in larger numbers. The CMD of I Zw 36 indeed contains quite a number stars of such red color, but many have high photometric errors.

In our discussion, we employ several sets of tracks. The first set is based on the "old" Padova tracks which are presently incorporated in our CMD simulator. Briefly, these tracks are based on the Padova library (e.g. Fagotto et al. 1994, Bertelli et al. 1994) and the stellar atmospheres of Bessell, Castelli \& Plez (1998). A "new" database of Padova tracks for low- and intermediate-mass stars recently became available (Girardi et al. 2000), and we considered these as well. We note that the changes were not so substantial that we felt compelled to replace the old tracks in our simulator. In Figure 9, we overplot onto the $\left[(\mathrm{J}-\mathrm{H})_{0}, \mathrm{H}_{0}\right] \mathrm{CMD}$ stellar evolutionary tracks from the "old" database. We show two sets of tracks with metallicities of $\mathrm{Z}=0.0004$ and $\mathrm{Z}=0.004\left(\mathrm{Z}_{\odot} / 50\right.$ and $\mathrm{Z}_{\odot} / 5$, respectively). The present-day ionized gas abundance of I Zw 36 is bracketed by these two. Tracks with $\mathrm{Z}=0.001$ (the closest match to the oxygen abundance in the database) fall inbetween the tracks of $Z=0.0004$ and $Z=0.004$, and so the tracks shown in Fig. 9 are a reasonable approximation for any stellar population which we might expect to find. The tracks do not extend to the extremely red colors observed for some of the asymptotic giants. This is due to the truncation at the first thermal pulse of the electronically available Padova tracks.

There are many indicators of present-day star-forming activity in I Zw 36 . The blue plume of the CMD of Fig. 9 is consistent with massive MS stars and BSGs. The MS turnoff of the $30 \mathrm{M}_{\odot}$ track is located well within the blue plume. Deharveng et al. (1994) suggest an age of less than $12 \mathrm{Myr}$ for the present star-forming event based on the stellar content observed in the FOC-UV images. Star formation in I Zw 36 must have been active within the last few Myr because it contains spectroscopically detected Wolf-Rayet stars (Izotov, Thuan \& Lipovetsky 1997). The UV spectrum indicates massive stars via their stellar wind 
lines, and the H-II regions further support the presence of ionizing, young and massive stars with ages of up to 10 Myr.

The red plume is the dominant feature of the I Zw $36 \mathrm{CMD}$ in the near-IR. It can be split into regions above and below the TRGB. The luminous portion of the red plume can in principle be composed of RSG stars and AGB stars. If we use the oxygen abundances in the ionized gas as an indication of the present-day metallicity and compare the upper CMD with the $\mathrm{Z}=0.001$ tracks, then clearly some of the stars in the upper red plume must be interpreted as RSGs. These stars have ages of a few ten Myr. RSGs were considered to be "missing" from the Deharveng et al. (1994) optical-near-UV CMDs, where the small number of red stars detected had large photometric errors and/or was accounted for by red leak and Galactic foreground contamination.

Comparing the two sets of tracks shown in Fig. 9 with the data illustrates a possible age-metallicity degeneracy for the luminous, red stars: we could interpret the few very high-luminosity stars at $M_{H}$ of about -10 to -9 and colors $(\mathrm{J}-\mathrm{H})_{0} \approx 0.9$ and 1.5 either as "high-metallicity" RSGs and thus, early descendants of massive stars, or as the TP-AGB phase of intermediate-mass stars of a "low-metallicity". In the latter case these stars would lie on extensions of intermediate-mass tracks beyond the first thermal pulse. However, we do not know whether a low-metallicity TP-AGB star would ever become luminous and red enough to match the most luminous and red stars in our data. As Fig. 12 suggests, there is one star classed AGB in I Zw 36 which is somewhat brighter than those classed AGB stars in VII Zw 403 and Mrk 178 (cf. also, Fig. 11 of SHCG00), and we should not attach too much weight to this singular data point. On the other hand, observations of AGB stars in the LMC suggest stars with $\mathrm{M}_{H} \approx-9.5$ are observed (Trams et al. 1999). If there are any AGB stars in the upper red plume, then their ages are over a hundred Myr.

We can more clearly separate the AGB from the RSG stars observationally when they 
are redder than $(\mathrm{J}-\mathrm{H})_{0} \approx 1.0$, which is also in the regime of the thermally pulsing, or TP-AGB phase. Indeed we see in Figs. 8 and 9 quite a few stars which are bright and red, $(\mathrm{J}-\mathrm{H})_{0} \approx 1.5$. TP-AGB stars in principle probe ages of a few hundred Myr to a few Gyr.

Stars below the TRGB are interpreted to consist in part of low-mass, red giants. Only low-metallicity sets of tracks can correctly describe the blue color of the red tangle; the $\mathrm{Z}=0.004$ tracks are too red. In other words, while the $\mathrm{Z}=0.004$ tracks can provide a fairly good description of the luminous stellar content of I Zw 36, they appear too red for the bulk of the RGB stars. This is similar to what we found for Mrk 178.

The comparison of the data with the tracks leads to the conclusion that we cannot describe the evolving stellar content with theoretical models of a single metallicity. From Fig. 9 it can be seen that the $\mathrm{Z}=0.004$ tracks have too red a $\mathrm{RGB}$, while the $\mathrm{Z}=0.0004$ have too blue an AGB location with respect to the data. The $\mathrm{Z}=0.001$ low-mass tracks can match the color of the RGB, but, like the $\mathrm{Z}=0.0004$ set, they also remain too blue with respect to the AGB stars. Thus, we could not find a single value for the metallicity which would simultaneously reproduce the colors of the old, the intermediate-age, and the young stars. The most obvious solution to achieve a better match is to allow some metallicity evolution with time in I Zw 36.

\subsection{The morphology}

The NIC2 camera was located on the highest-surface-brightness area of I Zw 36 as seen from the ground. Fig. 2 shows that the strongest star formation is centrally located on the chip. The near-IR-resolved stars and the gas are found to overlap spatially with the elongated plume of UV-detected massive stars and gas which crosses the FOC chip (see Fig. 1 of Deharveng et al. 1994). 
Figure 10 shows the NIC2 coordinates of the stars in the blue plume of our CMD, selected to have $(\mathrm{J}-\mathrm{H})_{0}<0.2$. Blue stars are resolved near the center and toward the lower right of NIC2, coinciding with the brightest H-II regions and the massive stars resolved in the UV. Our CMD, in addition to confirming the presence of young and massive stars, also exhibits the red tangle, that feature which contains intermediate- and low-mass stars and potentially harbors the truly ancient population of this galaxy. Fig. 10 illustrates how stars in the red tangle, which were selected to have $(\mathrm{J}-\mathrm{H})_{0}>0.5$ and $\mathrm{H}_{0}>23.3$, display a fairly uniform spatial distribution across the face of the galaxy (disregarding the "holes" in the center which are due to the high level of crowding in the star formation regions).

Surface-brightness profiles of I Zw 36 throughout and beyond the area of the galaxy encompassed by our NIC2 observations were investigated by Papaderos et al. (1996). They confirm I Zw 36 as a type "iE" BCD in the classification scheme of Loose \& Thuan (1995). In iEs, the faint outer isophotes are elliptical and the bright star formation regions are found in the vicinity of the center, although not necessarily at the exact center. As can be seen from Fig. 3a of Papaderos et al., the outer isophotes of I Zw 36 are quite extended. Papaderos et al. give surface-brightness profiles out to a distance of $40^{\prime \prime}$ from the center of the galaxy. Recall that the FOV of the NIC2 is about 19 arcsec across. The B-R color profile shows that I Zw 36 becomes redder with increasing distance from the center. As illustrated by Fig. 3e of Papaderos et al., the color first rises steeply outward from the core until a B-R color of about $1.0 \pm 0.1 \mathrm{mag}$ is achieved at a distance of about $20^{\prime \prime}$. At larger distances, the color remains constant at this level, until the profile is lost in the noise. The large color gradient in the inner $20^{\prime \prime}$ of the color profile has an obvious correspondence with the distribution of the resolved stars detected on NIC2 - luminous, blue stars dominate in the center, while faint red stars are seen everywhere.

We may age-date the stellar content at large distances (where the color gradient has 
flattened out), by comparing the B-R color to empirical cluster colors from Schmidt, Alloin \& Bica (1995). Such a comparison of course yields a luminosity averaged age. A B-R color of about $1.0 \pm 0.1 \mathrm{mag}$, lies between the cluster templates with age 0.7-2 Gyr $(\mathrm{B}-\mathrm{R}=0.91)$, and age 2-7 Gyr (B-R=1.17), or the dwarf Elliptical/ dwarf Spheroidal (dES) galaxy template $(\mathrm{B}-\mathrm{R}=1.17)$ of Schmidt, Alloin \& Bica. As an alternative, we also compared the B-R color to theoretical, spectral evolution synthesis models. The models by Bruzual \& Charlot (2000) indicate that, for a single stellar population ages from about 1 to 9 Gyr are possible, with lower ages corresponding to our high metallicity, $\mathrm{Z}=0.004$, and higher ages corresponding to our preferred, low metallicity, $\mathrm{Z}=0.0004$, assumption. Thus, both comparisons agree with our inference from the CMDs that stars with ages of at least 1-2 Gyr and probably much older, are present in I Zw 36. The faint red low surface brightness component of I Zw 36 can be traced to at least $40^{\prime \prime}$ from the center. At a minimum distance of 5.8 Mpc, the physical diameter of the low-surface brightness sheet of potentially ancient stars in I Zw 36 is thus at least $2.3 \mathrm{Kpc}$.

We previously illustrated, using the CMD of VII Zw 403, how small fractions of luminous young stars dominate integrated photometry even in the near-IR (see Table 2 of SHGC99). In H-band photometry of VII Zw 403, for instance, luminous RSGs (and AGB stars) amount to about $63 \%$ of the integrated light on the NIC2 chip. Origlia \& Oliva (1998) make use of this effect to estimate, for a sample of 35 BCDs, the mass involved in the burst relative to that in the old stellar component. They find that in most BCDs, including I Zw 36, the burst mass is much smaller than the mass of the older, underlying stellar population. These results confirm and expand on the earlier findings of Papaderos et al. (1996). Accordingly, the total luminosity of I Zw 36 is divided into about equal parts between the burst luminosity and that of the old component of the host galaxy (Papaderos et al. 1996). For any reasonable mass-to-light ratios for the two components, the old component must dominate the total stellar mass. We also found such a result using 
simulations of the near-IR CMD of Mrk 178 (SHGC00). Therefore, the stellar mass of most BCDs is apparently locked up in low-mass stars and not in the few massive stars which dominate the light. This raises the mass fraction of BCDs which must be attributed to baryons, although it is difficult to tell by how much (an attempt and a discussion of the pitfalls may be found in SHGC00).

We find from the comparison of the NIC2 data and the surface-brightness profiles, that I Zw 36 has a core-halo structure. The central core is defined by the star formation

regions; here the luminous stars dominate integrated photometry. Areas of the galaxy that fall outside of the NIC2 FOV, are red and of low-surface brightness. We interpret this to indicate that where the young, massive stars are absent, the integrated light comes from the intermediate- and/or low-mass stellar content of I Zw 36. Notice that prior to resolving stars in the background sheet with HST, it was not possible to be certain that the red color of the background sheet was due to RGB and AGB stars rather than to RSG stars. Since copious bright RGB stars first appear at an age of about 1 Gyr in populations of a wide range of metallicities (e.g., Sweigart, Greggio \& Renzini 1990), we quote this as our low age limit. The age estimate derived from the integrated B-R color at large distance is consistent with this minimum age. Both CMDs and integrated colors, in principle do allow larger ages for the oldest stars. However, age-metallicity degeneracy prevents us from claiming a specific age for the oldest stellar population in I Zw 36.

\subsection{The star formation history}

\subsubsection{The present-day SFR}

A quantitative SFR based on the IUE spectrum of I Zw 36 was derived by Fanelli, O'Connell \& Thuan (1988). The UV measures the fluxes of massive, newly-formed stars 
close to the maximum of their spectral energy distributions and is in principle one of the best gauges of SFR. In practice, the main drawbacks of UV-derived SFRs are their sensitivity to extinction and on the shape of the IMF. Fanelli, O'Connell \& Thuan combined two spectra of I Zw 36 taken through IUE's 10" x 20" aperture, which were slightly offset from one another, but basically centered on the brightest regions. They derive a SFR of $2 \times 10^{-3} \mathrm{M}_{\odot} \mathrm{yr}^{-1}$ from modeling the UV spectrum with a reasonably small extinction (i.e., not that which is indicated by the Balmer decrement). This SFR is for stars with masses between 2 and $100 \mathrm{M}_{\odot}$, assumes an IMF index of 2.8, and was scaled by them from their empirical measure involving only stars with masses above $10 \mathrm{M}_{\odot}$. If we adopt instead the Salpeter IMF (2.35) with limits of 0.1 and $100 \mathrm{M}_{\odot}$ (as is commonly used in dwarf galaxy research) then we find a rate of $1.7 \times 10^{-2} \mathrm{M}_{\odot} \mathrm{yr}^{-1}$. This rate is based on a distance of $4.6 \mathrm{Mpc}$; if we rescale to our minimum distance of $5.8 \mathrm{Mpc}$, then the SFR becomes $2.7 \times 10^{-2} \mathrm{M}_{\odot} \mathrm{yr}^{-1}$. Fanelli, O'Connell \& Thuan also provide an upper limit of $10 \mathrm{Myr}$ on the duration of the star-forming event seen in I Zw 36.

We derive a quantitative SFR directly from the massive stellar content observed on our CMD below. At our minimum distance the maximum rate which is consistent with the number of blue stars seen in the NIC2 field of view (about 19 square arcsec) is $2.5( \pm 0.4) \times 10^{-2} \mathrm{M}_{\odot} \mathrm{yr}^{-1}$. A principal concern with this CMD-based SFR is that it is constrained by a very small number of the most massive stars, only. The detection limits we achieve correspond to the main-sequence turnoff of a $15 \mathrm{M}_{\odot}$ star; and we are extrapolating rather a long way along the Salpeter IMF.

Other popular gauges of the SFR count the population of massive stars indirectly. For example, the nebular emission from gas ionized by massive stars is a widely used measure. In what follows, we determine the SFR using $\mathrm{H}_{\alpha}$ luminosity converted into SFR with the equation introduced by Hunter \& Gallagher (1986). We correct only for foreground 
extinction (using the galactic extinction from Schlegel, Finkenbeiner \& Davis (1998), with the Cardelli, Clayton \& Mathis (1989) extinction law), realizing that this yields a lower limit to the actual SFR. The spectrophotometric $\mathrm{H}_{\alpha}$ flux of Viallefond \& Thuan (1983) determined in a 6.' 1 circular aperture leads to a SFR of $1.9 \times 10^{-2} \mathrm{M}_{\odot} \mathrm{yr}^{-1}$ for I Zw 36 . As this flux was derived in an aperture which is small compared to the size of the galaxy, some fraction of the total flux from H-II regions as well as diffuse, ionized gas, might have been missed. A new value for the $\mathrm{H}_{\alpha}$ flux was recently measured by Hunter (2001). Using imaging observations, she obtains $1.29 \times 10^{-12} \mathrm{erg} \mathrm{cm}^{-2} \mathrm{~s}^{-1}$. This converts to a SFR of $3.8 \times 10^{-2} \mathrm{M}_{\odot} \mathrm{yr}^{-1}$. As discussed above, it is not clear how dust is distributed internally within I Zw 36. Therefore, we prefer not to correct the $\mathrm{H}_{\alpha}$ flux with the large internal extinction derived from the Balmer decrement. The uncertainty in how to apply an internal extinction correction is always a major limiting factor to the accuracy of $\mathrm{H}_{\alpha}$-derived SFRs.

The SFR can also be determined assuming that the radio continuum emission arises from synchrotron radiation produced by relativistic electrons that are accelerated when massive stars explode as supernovae (Condon 1992, but see Viallefond \& Thuan 1983). Radio fluxes suffer much less extinction than UV and optical ones. I Zw 36 was included in the NRVO/VLA Sky Survey (NVSS) and its total $1.4 \mathrm{GHz}$ flux is catalogued, $4.5 \mathrm{mJy}$ (Condon et al. 1998). We find a SFR of $2.2 \times 10^{-2} \mathrm{M}_{\odot} \mathrm{yr}^{-1}$ (with an extension of Condon's 1992 low-mass cut-off from $5 \mathrm{M}_{\odot}$ to $0.1 \mathrm{M}_{\odot}$ along the Salpeter IMF). The SFR from the radio continuum comes out to be smaller than the lower limit on the SFR derive from $\mathrm{H}_{\alpha}$ emission. This is a known effect. Cram et al. (1998) show that $\mathrm{H}_{\alpha}$ luminosities give systematically higher SFRs (by as much as an order of magnitude) than $1.4 \mathrm{GHz}$ luminosities for galaxies with small SFRs (smaller than about $10^{-1} \mathrm{M}_{\odot} \mathrm{yr}^{-1}$ ). The reason for this discrepancy has not yet been resolved.

We see that any and all methods available to determine the present-day SFR of I Zw 36 
have some kind of caveat. We adopt a SFR of $2.5 \times 10^{-2} \mathrm{M}_{\odot} \mathrm{yr}^{-1}$ as our best estimate.

\subsubsection{Comparing the near-UV and near-IR CMDs of I Zw 36}

The near-UV_optical CMDs of I Zw 36 by Deharveng et al. (1994) lack evolved red supergiants. This cannot imply a discontinuous SFH, since the near-IR CMDs show such a copious population of luminous red stars. To explain the absence of red supergiants on the near-UV - optical CMDs, we consider two observational biases: color bias and spatial bias.

To illustrate color bias, we reproduce in Figure 11 from Table 1 of Deharveng et al. (1994) the near-UV - optical [m342-m430, m430] CMD of I Zw 36, and compare it with our $\left[(\mathrm{J}-\mathrm{H})_{0}, \mathrm{H}_{0}\right]$ CMD. The near-UV_optical CMD of I Zw 36 approximates U-B. It exhibits a strong blue plume from the present generation of massive stars. Deharveng et al. age-date the young generation of stars at $\leq 12 \mathrm{Myr}$ (but do not give a SFR). A few stars are observed in the red plume. Deharveng et al. discuss these stars explicitly. They find that most of them are not real owing to red leak, large error bars, and the possibility of foreground contamination. They conclude that no, or very few, RSGs exist in I Zw 36. This supports their burst age. However, since the integrated color of I Zw 36 is redder than would result from just the ongoing star formation, Deharveng et al. propose the existence of an underlying background sheet of much older stars. This is consistent with a SFH that had a long quiescent period prior to the current event. By contrast, the same region of I Zw 36 observed in the near-IR reveals a strong component of luminous, red stars. These are in part the "missing" RSGs. The detection of copious AGB stars in particular extends the star formation of I Zw 36 to times that pre-date the event seen in the near-UV-optical CMD. These stars suggest a more or less continuous history of star formation over the last Gyr. In essence, the near-UV - optical CMD is best used to derive the stellar content for the past $10 \mathrm{Myr}$, while the near-IR CMD yields a census for the past several $100 \mathrm{Myr}$. 
Spatial biasing could also have affected the Deharveng et al. results. As we now know, the stellar content of BCDs varies as a function of position. Fig. 1 illustrates that the NIC2 and FOC pointed to roughly the same region in the star-forming core of I Zw 36 . However, massive, young stars completely filled the small FOV of the FOC, possibly masking faint red stars due to crowding (compare Fig. 10). Their images also exhibit a large contribution by ionized gas. This possibly made stellar detections difficult for DAOPHOT, since it expects a flat background and does not perform well in the presence of a spatially nonuniform background (Stetson, Davis, \& Crabtree 1990).

In summary, this section serves as a note of caution that SFHs derived from CMDs may be affected by observational biases which, though they can in part be addressed with

simulated CMDs, may prevent us from accurately recovering in all cases the actual SFH of a galaxy. It also serves to show that any gaps on CMDs need to be carefully scrutinized for observational effects before concluding a discontinuous SFH.

\subsubsection{Intercomparison of the near-IR CMDs of BCDs}

Near-IR photometry to deep limiting magnitudes is still a new technique for stellar population studies. In this section, we compare the near-IR CMD of I Zw 36 with that of two nearby BCDs, VII Zw 403 and Mrk 178, and with that of I Zw 18.

We previously resolved VII Zw 403 and Mrk 178 in the near-IR to such deep limiting magnitudes that we were able to classify their stellar content down to magnitudes below the TRGB. This classification scheme was originally developed by comparing the relatively well-understood V, I CMD of VII Zw 403 with the locations of the same stars on its J, H CMD (SHGC99). We used this classification to investigate the J, H CMD of Mrk 178 in SHGC00. In Fig. 12, we apply the classification to I Zw 36. We note that these three 
galaxies are all relatively nearby and that the data have similar crowding and blending properties. (In Fig. 12, the red tangle of VII Zw 403 extends to blue colors, and the blue plume extends into the red tangle, at the faintest magnitude levels. This is due to the fact that the near-IR data have different errors than the optical data from which the classification was derived. While we place the blue vs. red plume dividing line at 0.5 mag in Fig. 12, we plotted stars with $(\mathrm{J}-\mathrm{H})_{0}<0.2$ only as blue plume of I Zw 36 in Fig. 10, to safely split off the blue plume from the red tangle.)

In Fig. 12, we color code all stars with $(\mathrm{J}-\mathrm{H})_{0}$ color smaller than 0.5 in blue. The purpose is to illustrate that we consider these stars to be part of the blue plume. Stars with colors $0.5<(\mathrm{J}-\mathrm{H})_{0}<0.85$ and $\mathrm{H}_{0}$ above the TRGB are shown as RSGs in magenta. Stars with colors $>0.85$ are AGB stars and shown in black. This coding combines the early, or E-AGB, and the TP-AGB phases. Note that stars in the TP-AGB phase are expected to have $(\mathrm{J}-\mathrm{H})_{0}>1$. The remaining stars are color-coded in red and considered to belong to the red tangle.

Comparing the stellar content of VII Zw 403, Mrk 178, and I Zw 36 in this way, we find a great deal of similarity. All the major phases of stellar evolution are represented on the CMD. In SHGC00 we presented a set of exemplary CMD simulations in the near-IR for Mrk 178. (We note that in order to avoid color biasing, the present-day SFR was derived from the $\mathrm{H}_{\alpha}$ luminosity as well as from simulated CMDs.) Since the near-IR CMD of I Zw 36 exhibits the same morphology as that of Mrk 178, the star formation histories are similar. In particular, the strong AGB stellar component suggests that star formation was very active in the recent past, i.e., a few hundred Myr ago. A similar result, enhanced star formation in the interval 600-800 Myr, was gleaned from simulations of the optical CMD of VII Zw 403 (Lynds et al. 1998).

The famous I Zw 18, a galaxy still hotly contested to be forming its first stars in the 
present burst, was observed with HST/NIC2 by Östlin (2000). Its near-IR CMD is difficult to compare to that of I $\mathrm{Zw} 36$. The CMD contains only about 70 stars, compared to over 700 stars in I Zw 36. Therefore, the stochastic properties of the two datasets are quite different. I Zw 18 is at about twice the distance of I Zw 36 (Östlin adopts $12.6 \mathrm{Mpc}$ ). Owing to the larger distance of I Zw 18, its NIC2 data have different blending and crowding properties than those of I Zw 36. Östlin infers a more or less continous star formation over the last 1 Gyr; a SFR peak at 14 Myr is identified with the current starburst. The optical, HST/PC2 CMD of I Zw 18 was previously modeled by Aloisi, Tosi \& Greggio (1999), who adopted a distance of $10 \mathrm{Mpc}$. From the comparison of observed and synthetic luminosity functions they find a single starburst with a duration of order $10 \mathrm{Myr}$ is definitely ruled out. However, a single episode can reproduce the data if it started more than 200 Myr ago and either continues today or stopped 5 Myr ago. A two-event scenario is also possible and preferred by Aloisi, Tosi \& Greggio, with a first episode from 1 Gyr to 30 Myr ago at constant SFR, and a second one, 7-10 times stronger, from 20 to 15 Myr ago. In spite of the ample amount of HST time devoted to observations of I Zw 18, the conclusions about its recent $\mathrm{SFH}$ are still ambigous and await, in particular, a distance determination based on a stellar distance indicator.

To summarize, we find that the SFH of I Zw 36 can be well traced over a wide range of ages with the aid of near-IR CMDs. There do not appear to have been any distinct, strong bursts and long quiescent periods. This conclusion is further supported by our models of the stellar distribution on the CMD, presented below.

\subsubsection{The mode of star formation}

We now turn to the question of what exactly is the star formation mode of nearby BCDs. Stars are distributed across the major areas of the CMDs of VII Zw 403, Mrk 178, 
and I Zw 36. They represent all the stellar masses and major stellar evolutionary phases which are above the detection limits. There are no obvious gaps in the distribution of stars. Gaps on the CMDs are expected for BCDs; their star formation is commonly viewed as episodic. For instance, Mas-Hesse \& Kunth (1999) find a very short mean duration of 3.5 Myr for the starbursts in a sample of BCDs and dwarf Irregular (dIrr) galaxies, while Thuan et al. (1999) envision quiescent periods of 2-3 Gyrs between the active starburst phases of BCDs. This mode would accomodate a few bursts over the Hubble time, and prevent too much chemical enrichment and gas consumption.

To investigate the possibility that gaps in the SFH are hidden in our data, we simulated a large number of synthetic CMDs. We used the simulator introduced in SHGC00, which evolved from the code of Tosi et al. (1991) adapted to HST data by Greggio et al. (1998). In brief, the simulator is based on the evolutionary tracks of Fagotto et al. (1994), and the tables of Origlia \& Leitherer (2000) for bolometric corrections. The simulations in this paper were carried out with an improved version of the simulator described in SHGC00, leading to a better description of the photometric errors. The error distributions and completeness fractions in the simulator represent very well the I Zw 36 data.

Being able to trace resolved stars with ages of at least 1 Gyr on the CMD, we know that I Zw 36 is not a "primeval" galaxy. Lacking distinct main-sequence turn-offs of an old stellar population (the data are not very deep as I Zw 36 is quite distant), we cannot tell the age of formation of the first stars. In what follows, we therefore discuss cases in which the galaxy is modeled to be "young", as well as cases in which the galaxy is considered to be "old". We show what kind of models give good matches to the data, by comparing the models to the data qualitatively, via CMD morphology, and quantitatively, via luminosity functions (LF). 


\section{Young-galaxy models}

Several exemplary, synthetic CMDs are provided in Fig. 13. For this particular set of examples, we used our low-metallicity $(\mathrm{Z}=0.0004)$ simulator, with the appropriate, short distance modulus. We also adopted the Salpeter initial mass function. Notice that the simulator does not place TP-AGB stars on the CMD, as we do not have stellar parameters for this phase. As in SHGC00, we keep track of potential TP-AGB stars in a counter. We use the best available constraints on the masses of stars which evolve into the TP-AGB phase $\left(1.5\right.$ to $\left.3 \mathrm{M}_{\odot}\right)$, and the lifetime of the TP-AGB phase (1.5 Myr), yet our simulations tend to always produce more synthetic stars in the TP-AGB phase than are actually observed.

The simulations displayed in Fig. 13 consist of five examples. Case (a) shows the CMD morphology for continuous star formation that started 2 Gyr ago. All other panels illustrate a short, 10-Myr long starburst, preceded by a widening gap in star formation, preceded by the formation of the remaining stars with ages up to 2 Gyr. The gap lasts 10 Myr for case (b), $100 \mathrm{Myr}$ for (c), $500 \mathrm{Myr}$ for (d), and $1 \mathrm{Gyr}$ for (e). The star formation was completely stopped in the gaps, that is, the SFR was dropped to $0 \mathrm{M}_{\odot} \mathrm{yr}^{-1}$ for the duration of the quiescent period.

Notice how cases (a), (b), and (c) all produce acceptable CMD morphologies as compared with the data. Cases (d) and (e), where star formation is interrupted for several hundred Myr, exhibit a clear lack of RSG/AGB stars. In other words, the red plume so well-populated in the data is cut away by the cessation of star formation. This indicates that gaps of several hundred Myr in recent history are disallowed by the data.

It is difficult to fine-tune the gap simulations and to derive more precise gap durations, time placements, and amplitudes. For one, the time resolution of the data is not uniform across the CMD. The highest resolution occurs in most recent times. Another issue is the 
empirical adjustment of the SFR (and/or metallicity) on either side of the gap. Finally, dropping the SFR to $0 \mathrm{M}_{\odot} \mathrm{yr}^{-1}$ in the gap is an extreme assumption, which may not be realized in nature. While we cannot explore the entire parameter space of possible gap durations, we can nontheless make some general statements as to what probably is, and what is not, compatible with the data.

All of the available data from the UV through the radio show evidence of massive stars. Therefore, we cannot drop the SFR in the recent $10 \mathrm{Myr}$ (or $100 \mathrm{Myr}$, etc.) down to zero. Such a gap is not allowed. However, if we slide the 10 Myr gap back by a mere $10 \mathrm{Myr}$ in the simulator (case (b)), we already lose it in the data. As the time resolution decreases with age, we could also hide a $10 \mathrm{Myr}$ gap on the CMD anywhere else at earlier times. Similarly, since we can hide a 100 Myr gap between 10 and 110 Myr (case (c)), where time resolution is high, we can also introduce it at earlier times and it would go unnoticed in the data. We feel confident that we can rule out discontinuities in the SFR of the order of several 100 Myr, within at least the recent Gyr. In addition to the simulation shown as case (d), we experimented with a $500 \mathrm{Myr}$ gap that we slid back in time within the recent Gyr. We find that a $500 \mathrm{Myr}$ gap is too long to be hidden by the data. We conclude that somewhere between 100 and $500 \mathrm{Myr}$, we transition to a gap length (for a zero SFR gap) that cannot be hidden anywhere in the recent Gyr. Finally, it is also impossible to stop the star formation for more than a Gyr in the recent 2 Gyr. If this gap occured in the most recent Gyr, it would push about $90 \%$ of the stellar content into the red tangle below the TRGB, something that clearly disagrees with the data (case (e)). Even placed elsewhere, such a long gap clearly unbalances the morphological appearance of the CMD. In general, the red tangle area of a CMD provides virtually no age resolution. It can in principle contain stars with ages similar to the Hubble time. We can hide Gyr-long gaps here, or drop the SFR to more realistic "low states" for extended periods of time, and we have no way of noticing. 
We now provide some details on how we ran the simulations, and on the SFRs which ensued. When we assume continuous star formation which started 2 Gyr ago (case (a)), then the SFR implied by our CMD is typicially about $4.9( \pm 0.3) \times 10^{-3} \mathrm{M}_{\odot} \mathrm{yr}^{-1}$. The error reflects the statistical variations that come about when we run a large number of simulations with identical parameters. The 2-Gyr-constant-SFR model is qualitatively a good match of the CMD, and quantitatively a good match to the LF. This is illustrated in Fig. 14, where we compare the LF of the data with the LF of a 2-Gyr-continuous SFR model. The data and the model were binned in 0.25 mag. intervals, and the errorbars shown are \pm the squareroot of the starcount in each bin. Notice in particular that it was possible to match the observed power at the TRGB (where we have a large starcount) to within the respective errors. In a large set of simulations, some individual simulations showed deviations at the TRGB; these were smaller than $20 \%$ and in the direction of generating too many stars just above the TRGB and too few just below the TRGB.

Between about $50-100 \%$ of the stars in the blue plume could potentially be massive stars. If all of the stars in the blue plume are indeed massive MS stars and BSGs with ages no larger than $10 \mathrm{Myr}$, then there is a high power in the ongoing event. To simulate and test the burst+quiescent scenario, we indeed put all of the power consistent with the number of stars in the blue plume (i.e., $10 \%$ of all stars on the CMD) into the young burst. (An alternative, discussed below, is to assume that the ongoing event started earlier than 10 Myr ago, and that some of the stars at the bottom of the blue plume should be interpreted as BL stars.) We adopted 10 Myr for the duration of the event, since this is typical of the length associated with the starburst in I Zw 36 by Fanelli, O'Connell \& Thuan (1988), $\leq 10$ Myr, and Deharveng et al. (1994), $\leq 12$ Myr. With these assumptions, the present-day star formation rate indicated by the blue plume of the NIC2 CMD is $2.5( \pm 0.4) \times 10^{-2} \mathrm{M}_{\odot} \mathrm{yr}^{-1}$, or about a factor of 5 up from continuous star formation over the last 2 Gyr. The error on the SFR again reflects stochastic variations, and was derived by running a large number 
of simulations with the above parameters. For each of the cases (b) through (e) shown in Fig. 13, we used a "fresh" simulation for the 10-Myr starburst, allowing us to illustrate the statistical effects in the supergiant population (cf. Greggio 1986). The SFR given above is the highest rate that is consistent with the number of observed stars. Cases using the maximum SFR can produce satisfying synthetic CMDs and LFs. For example, we achieved an excellent match to the data with a model that has a high-power starburst preceded by a 100 Myr gap. In this model, the SFR in the 10-Myr burst was $2.1 \times 10^{-2} \mathrm{M}_{\odot} \mathrm{yr}^{-1}$, and the SFR from 110 Myr to 2 Gyr was $4.4 \times 10^{-3} \mathrm{M}_{\odot} \mathrm{yr}^{-1}$. This implies that solutions in which the ongoing event is very short and by about a factor of 5 stronger than the average recent SFR are allowed.

If we assume instead that fewer than $100 \%$ of stars in the blue plume are in reality massive, young stars born in the recent $10 \mathrm{Myr}$, and allow a longer duration for the ongoing event, then the SFR goes down. The duration of the ongoing event is not constrained by our data. Recall that a 2-Gyr-continuous SFR model matches the CMD and the LF of the data. If the star formation extended to earlier times, then some of the stars at the bottom of the blue plume would be interpreted as older, BL stars at the blue edges of their blue loops (cf. Fig. 12 of SHGC00). Since there are not a lot of stars in the blue plume in total, we can cut the SFR derived for the 10-Myr-burst scenario and still easily make up for the few missing faint stars with a contribution by BL stars.

To summarize, the near-IR CMD does not constrain well a recent, short star-forming event. We can accomodate either a long episode at a relatively low rate, or allow for a very recent SFR enhancement, of virtually any factor up to 5. The maximum current SFR compatible with our CMD is the same as the one derived from UV and radio data. 


\section{Old-galaxy models}

So far, we have treated I Zw 36 as a young galaxy with no star formation earlier than 2 Gyr ago. We emphasize that a young model galaxy with a more or less continuous SFR can provide a good match of the data. The young model is our conservative model, since we cannot really proove the existence of ancient stars with the CMDs in hand.

However, we have circumstantial evidence which indicates I Zw 36 may be an old galaxy. We know that stellar evolutionary tracks for low masses and hence, old ages, do pass through the red tangle of the CMD. We also know that the integrated color of the background sheet at large distances from the star-forming core can be identified with an old stellar component. Therefore, we also ran several simulations which extended the SFH further back than 2 Gyr (to 5, 7, and 15 Gyr).

These simulations are constrained by the fact that we always need to produce 712 survivors on the CMD. Therefore, in general, the older the stellar population, the lower the SFR. We show in Fig. 14 the LF of a simulation for a 15-Gyr-continuous SFR model. We see that this simulation has too much power at the TRGB, and produces too few stars of intermediate and high luminosity above the TRGB. This implies that if we assume that I Zw 36 is an old galaxy, then some amplitude variations in SFR are required on long time scales. Specifically, for ages larger than 2 Gyr, we need more power in the recent history, and less power in ancient history, than what is provided by models with continuous SFRs.

We therefore explored simulations in which we assumed that the galaxy is old, and in which we adjusted the recent and early SFRs separately to match the CMD and the LF. We define as the recent SFR the SFR between 0 and 1 Gyr, and as the early SFR the SFR from 1 to 15 Gyr. This allows us to derive constraints on the ratio of $\mathrm{SFR} /<\mathrm{SFR}>_{\text {past }}$, the birthrate parameter, directly from the resolved stellar content. 
A natural dividing line between SFR and $<$ SFR $>_{\text {past }}$ occurs indeed at an age of 1 Gyr since at this age, RGB stars start to populate CMDs at the TRGB in large numbers. We used the technique of the SG and AGB boxes developed in SHGC00. We assigned all stars counted within these boxes to the recent Gyr. Specifically, this part of the model has a constant star formation which started 1 Gyr ago and is constrained by having to yield $20 \%$ of its survivors with magnitudes $\mathrm{H}_{0}<23.3$ and colors $-0.5<(\mathrm{J}-\mathrm{H})_{0}<1.0$. When simulations are run, they bring along also the progenitors of theses stars which are below the TRGB. We then assumed that the remaining stars below the TRGB not yet accounted for have ages between 1 and 15 Gyr, and simulated them in the second part of the modelling process. In this way, we are able to estimate the contrast between SFR and $<\mathrm{SFR}>_{\text {past }}$ from the CMD with a reasonable set of assumptions. (Refer to SHGC00 for examples of how to hide variations in the SFR among the early SFH of a BCD, also for comments on how these affect the total astrated mass of a galaxy.)

The LF of a model from our run of 14+1-Gyr-continuous scenarios is shown in Fig. 14. We find that such models can provide good matches of the CMD and the LF of the data, with a quality that equals the 2-Gyr-continuous models. These simulations yield a recent SFR which is $5.7( \pm 0.8) \times 10^{-3} \mathrm{M}_{\odot} \mathrm{yr}^{-1}$; and an early $<\mathrm{SFR}>_{\text {past }}$ which is $4.3( \pm 0.6) \times 10^{-4} \mathrm{M}_{\odot} \mathrm{yr}^{-1}$. The ratio $\mathrm{SFR} /<\mathrm{SFR}>_{\text {past }}$ is about a factor of 13 . The estimated factor of 13 for I Zw 36 is very similar to our result for Mrk 178 (SHGC00), where $\mathrm{SFR} /<\mathrm{SFR}>_{\text {past }}$ is 8-12 depending on the assumed metallicity.

To summarize, models which match the data can be obtained by extending the SFH to old ages, as long as the power in the young and old stellar content are adjusted appropriately. Being sensitive to SFR in the recent $1 \mathrm{Gyr}$, these CMD simulations are constrained by the power consistent with star formation in the recent Gyr, and leave the remaining stars to be assigned to any potential old $\mathrm{SFH}$. In general, $\mathrm{SFR} /<\mathrm{SFR}>_{\text {past }}$ 
increases from 1 when the initial time of star formation is 2 Gyr, to an order of magnitude when the initial time is $15 \mathrm{Gyr}$. The trend basically reflects the increase in the temporal range over which the star formation is demanded to produce the observed number of stars below the TRGB. Both kinds of scenarios, young and old, match the CMD and LF of the resolved stars. The morphology and integrated colors of the background sheet lead us to prefer an old galaxy over a young galaxy.

\section{Modelling summary}

This case study of I Zw 36 allows us to gain insight into the star formation mode of BCDs. Because I Zw 36 is the most distant BCD in our NICMOS survey, its data have the largest errors and incompleteness fractions. Gaps would be easier to hide in this data set, than in the CMDs of VII Zw 403 and Mrk 178. As explained above, I Zw 18 is at such a much larger distance that its near-IR CMD is not directly comparable with the I Zw 36 data or simulations.

A model which assumes a single, short burst preceded by a long quiescent period does not match the observations. Our conclusions are consistent with those reached by Aloisi, Tosi \& Greggio (1999) for the SFH of I Zw 18 based on models of its optical CMD. This causes somewhat of a paradigm shift in the way we view BCDs. Scenarios which consider current BCD activity as several-orders-of-magnitude strong "delta functions" in the SFR do not give an accurate record of what we see on CMDs.

Good models of the observed CMDs can be produced with more-or-less continuous SFRs in recent times. The recent mode of star formation of BCDs was therefore either continuous with modulations by factors of a few (thus allowing for some "burstiness"), and/or "gasping" in the terminology of Tosi (1998). In particular, however, in all of the BCDs resolved with HST, star formation is seen to have been active for at least 1 Gyr. 
BCDs are unlikely to be truly primeval galaxies, although they may be fairly young.

We consider the present activity part of an extended high state. Extended periods of low states in SFR may have occured at early times, if BCDs are old galaxies, but deeper single-star photometry is needed before we can derive the age of formation of BCDs. The CMDs allow us to nevertheless constrain the average powers in the high and low states. For instance, we may assume that BCDs are ancient galaxies, having formed their first stars as much as 15 Gyr ago. If this is the case, then we derive a factor of about 10 for $\mathrm{SFR} /<\mathrm{SFR}>_{\text {past }}$ for I Zw 36, similar to what we inferred in SHGC00 for Mrk 178.

\section{Discussion}

I Zw 36 is observed with HST to deep enough limiting magnitudes to resolve stars in the RGB phase. We consider it our most important finding that whenever deep enough observations are available, we discover RGB stars in BCDs. These stars bear witness to star formation which predates the ongoing activity. The RGB stars can in principle be much older than the minimum age of 1-2 Gyr that we are able to assign to them based on our CMDs. However, we require even deeper CMDs able of resolving stars on the blue horizontal branch or an old main-sequence turn-off, before we can ascertain the presence of truly ancient (>10 Gyr) stars. Such data are beyond the observing capabilities of the present time. Nevertheless, we think that the combination of resolved RGB stars together with a low-surface-brightness, red halo, makes a strong case for an underlying population of old stars in I Zw 36.

Three BCDs have now been studied well below the TRGB with NICMOS: VII Zw 403,

Mrk 178, and I Zw 36. They range in metallicity (O abundance of the ionized gas) from $\mathrm{Z}_{\odot} / 10$ to $\mathrm{Z}_{\odot} / 20$. They are on the low-metallicity side of the observed peak in O-abundance 
distribution of BCDs (e.g., Izotov \& Thuan 1999). It is this low O abundance which has traditionally been the driver for the youth hypothesis. In the alternative hypothesis, assuming BCDs are old galaxies, they should have had only a few, short starbursts separated by long quiescence periods, else they enrich too quickly and/or run out of gas (see, e.g., the review of Thuan 1991). The old galaxy hypothesis is favored by our finding of a red backgound sheet which extends far beyond the core of star-forming activity.

What then, are the strengths and duty-cycles of BCD starbursts? BCDs are often thought of as intensely star-forming systems. However, the recent star formation rates of VII Zw 403, Mrk 178, I Zw 36, and I Zw 18 are only several $10^{-3}$ to a few $10^{-2} \mathrm{M}_{\odot} \mathrm{yr}^{-1}$. The star formation rates of these BCDs are quite moderate. Given moderate star formation rates, these BCDs should not have any difficulty economizing their gas supply for more than another to up to several Gyr.

More specifically, the model SFRs derived for I Zw 36 shed some light on its gas-consumption time. At a high SFR, $2.5 \times 10^{-2} \mathrm{M}_{\odot} \mathrm{yr}^{-1}$, consistent with a 10-Myr burst, and assuming $100 \%$ efficiency, the available $6 \times 10^{7} \mathrm{M}_{\odot}$ of $\mathrm{H}-\mathrm{I}$ gas will be consumed in about 2.5 Gyr. However, it is clear that such a burst has a short duration. The average SFR over the most recent Gyr is $5.7 \times 10^{-3} \mathrm{M}_{\odot} \mathrm{yr}^{-1}$. If I Zw 36 continues to make stars at this average rate, its H-I supply will last for another 10 Gyr. In other words, excessively rapid gas consumption does not have to be taking place, and thus does not force us to adopt episodic star formation with short bursts and long quiescent periods as the star formation mode of BCDs (although one can envision that all gas available for star formation will eventually be used up). Finally, if we assume a large formation age, e.g., of 15 Gyr, then the $<$ SFR $>_{\text {past }}$ consistent with the red tangle area of the CMD is an order of magnitude below that of the current high state, implying a gas-consumption time much longer than a Hubble time. Thus an earlier, low state of star formation insures that BCDs are gas rich 
(and metal-poor) in the present epoch.

Simulations of the CMDs of VII Zw 403, Mrk 178, I Zw 36 and I Zw 18 have shown that star formation is consistent with a more or less continuous history at least over the past Gyr. This is what we call the high state. Fig. 13 illustrates how continuous star formation is that mode which best represents the near-IR CMD morphology for I Zw 36, although interruptions in star formation can be supported by the data, as can be short bursts if they have less than about an order of magnitude in power compared to the recent average rate. Whereas BCDs as a class have been viewed as systems undergoing short and strong starbursts separated by long quiescent periods (e.g., Searle, Sargent \& Bagnuolo 1973; Thuan 1991; Mas-Hesse \& Kunth 1999), our observations indicate that such behavior may not be the norm.

The BCDs I Zw 36, Mrk 178, and VII Zw 403 belong to the iE type, which comprises $70 \%$ of all BCDs. If they are representative of the iE types as a whole, then it stands to reason that indeed the majority of BCDs do not undergo short bursts of star formation separated by Gyr-long periods of quiescence, as has been our common conception of the star formation histories of BCDs. I Zw 18 belongs to the rare few percent of BCDs which are still considered to be candidate primeval galaxies, although there is much controversy on its age of formation. While its optical and near-IR CMDs clearly indicate that star formation began at least a Gyr ago, if not earlier, the results for the mode of star formation are not as yet conclusive.

We cannot comment on the SFH past the "1-2 Gyr frontier" of the CMDs for any BCD. However, for I Zw 36 and Mrk 178, we provide an estimate of SFR $/<$ SFR $>_{\text {past }}$ based on resolved stellar content. If we assume that these galaxies are as old as 15 Gyr, then the contrast between the present high state and the past low state is as high as about an order of magnitude. The possibility of extended star formation will require us to look for 
ways in which we can reconcile the low $\mathrm{O}$ abundances seen in the ionized interstellar gas of BCDs with a more or less continuous history of star formation. This may be possible if star formation rates in early and extended low states are small enough (Legrand et al. 2000; Legrand 2000; Henry, Edmunds \& Köppen 2000). Indeed our constraints for I Zw 36 and Mrk 178 give low states (for an age of $15 \mathrm{Gyr}$ ) of a few times $10^{-4} \mathrm{M}_{\odot} \mathrm{yr}^{-1}$, similar to what Legrand's model assumes for the early SFH of I Zw 18.

The nature of our data does not allow us to provide insight on details of the SFR early in the history of BCDs (see SFGC00). In order to achieve that, CMDs with much deeper limiting magnitudes are needed. We note, however, that even among the 20 or so dwarfs of the Local Group studied to deep limiting magnitudes (see the recent review of Grebel 2000), an episodic mode of star formation is the exception rather than the rule. Among the Local Group dwarfs, the Carina dSph is presently the only convincing case of a dwarf galaxy which experienced several distinct episodes of star formation separated by Gyr-long quiescent periods (Smecker-Hane et al. 1994; Hurley-Keller, Mateo \& Nemec 1998). Since episodic star formation is so rare in Local Group dwarf galaxies, be they dIrr, dwarf Spheroidal (dSph), or dIrr/dSph in type, it is perhaps not surprising that it appears to be uncommon among the BCDs.

We have been interested in investigating the extent to which the SFHs of BCDs are compatible with models of faint blue excess galaxies. For instance, BCDs could provide alternative remnants for the delayed-formation scenario of dwarfs. This scenario attributes faint blue excess galaxies to a population of bursting, blue dwarfs at intermediate redshift which has, by and large, faded and/or merged by the present epoch (e.g. Babul \& Ferguson 1996). Today's dSph galaxies have been considered front runners for the faded, red remnants (e.g. Guzmán et al. 1998). However, galaxy counts in the Hubble Deep Field (HDF) have demonstrated that models in which star formation takes place in a single, short 
burst followed by fading, overproduces faint red remnants with respect to the observed counts (Ferguson \& Babul 1998). We have considered that BCDs could provide some of the remaining, and recently "rejuventated", remnants of faint blue galaxies. Greggio et al. (1998) investigated the dIrr galaxy NGC 1569, and concluded that its stellar content is consistent with the kind of starburst required in the Babul \& Ferguson model. Aloisi, Tosi \& Greggio find that the SFR of I Zw 18 falls short by 2 orders of magnitude to make it a local counterpart of the faint blue galaxies according to the Babul \& Ferguson model. We tested the Babul \& Ferguson scenario on the CMD of Mrk 178, and found that it was difficult but not impossible to achieve a burst of the necessary strength among the RGB population (SHGC00). The general lack of a highly episodic nature of the star-formation rates of local dwarf galaxies, including BCDs, would seem to speak against the scenario in which the faint blue galaxies are briefly bursting dwarfs.

The evidence which accumulated over the past few years now suggests that neither whole-sale merging of dwarfs (Rocca-Volmerange \& Guideroni 1990), nor strong luminosity evolution in dwarfs (Phillips \& Driver 1995), nor a population of blown-away dSphs (Babul \& Rees 1992), can account for all aspects of the faint blue galaxy excess. If BCDs are predominantly galaxies with gentle star formation variations, then a steeper faint end of the local, optical galaxy luminosity function (Gronwall \& Koo 1995) may provide the most natural explanation of the faint blue excess galaxies.

Extended periods of activity would also help to explain why three decades of work have not revealed any obvious candidates for BCDs in quiescence in the local Universe (Kunth \& Östlin 2000). 


\section{Conclusions}

I Zw 36 is the third low-metallicity BCD of the $\mathrm{iE}$ subtype in which the RGB is revealed with HST/NICMOS. The data indicate stars with ages of at least 1-2 Gyr, and a few lines of evidence hint at an even earlier age of formation. The ongoing star formation in the galaxy core, including Wolf-Rayet stars, occurs within an extended body of older stars. These low-mass stars make the dominant contribution to the stellar mass of the galaxy.

We emphasize the usefulness of near-IR CMDs in detecting the evolved descendants of intermediate and low-mass stars whose spectral energy distributions peak in the near-IR. Thus, near-IR CMDs complement near-UV CMDs, which preferentially sample the young populations of hot, massive stars; they help to avoid the problems of color biasing.

While I Zw 36 is actively forming stars in its core, the present-day star formation rate is not very high. At its present rate of $2.5 \times 10^{-2} \mathrm{M}_{\odot} \mathrm{yr}^{-1}$, and assuming a $100 \%$ efficiency, I Zw 36 will run through its $\mathrm{H}-\mathrm{I}$ supply of $6 \times 10^{7} \mathrm{M}_{\odot}$ in about 2.5 Gyr. The limited gas-consumption time has been one of the reasons why star formation in BCDs is considered to proceed in a series of a few short bursts separated by long quiescent periods. An alternative to this extreme scenario are more extended high and low states of star formation. The average SFR which is consistent with the high activity over the recent Gyr, for example, can continue for another 10 Gyr before it consumes all of the H-I gas.

We address the mode of star formation of BCDs with simulated CMDs. As a case study, we provide simulated star-formation histories of I Zw 36 . We show that if we allow short stops $(10-100 \mathrm{Myr})$ in its recent star formation history we can still produce CMDs which match the data. However, once star formation is interrupted by several $100 \mathrm{Myr}$, the morphology of the CMD changes so drastically that we conclude long gaps with zero star formation cannot be hidden in the data. We find for all three iE BCDs investigated by our team not only that the present star formation does not represent the first event in their 
history, but also that star formation has not proceeded, at least in recent times, in a series of short, strong bursts with long quiescent periods. A moderate modulation in the recent SFR is consistent with our data. We also gleaned some information about possible, ancient star formation from the red giants of I Zw 36. Assuming an age of 15 Gyr, we divided the SFH into the interval from 0-1 Gyr, and from 1-15 Gyr; and we derived SFR $/<\mathrm{SFR}>{ }_{\text {past }}$ $=13$. If I $\mathrm{Zw} 36$ is indeed an ancient galaxy, then the SFR in the recent Gyr implied by its CMD is by as much as an order of magnitude higher than its average past SFR, in the region encompassed by the field of view of the NIC2 detector. We previously determined a similar birthrate for Mrk 178.

Extended periods of high- and low-states of star formation, which we infer from model CMDs to be viable SFHs, would alleviate the problem of the missing quiescent counterparts of BCDs in the local Universe, and have implications concerning models in which faint blue excess galaxies are bursting dwarfs. They also challenge star formation histories of BCDs based on chemical-evolution modelling, by allowing for the existence of gas-rich and metal-poor galaxies which are nevertheless not young.

We thank Dr. Deidre Hunter for sharing the $\mathrm{H}_{\alpha}$ flux of I Zw 36 prior to publication. We also thank Dr. Andrew Hopkins for help in extracting and interpreting the radio data. Work on this project was supported through HST grants to RSL and MMC (projects 7859 and 8012). UH acknowledges financial support from SFB 375. LG acknowledges support from the Alexander von Humboldt Stiftung. We made extensive use of the SIMBAD and NED data bases. 


\section{REFERENCES}

Aloisi, A., Tosi, M., Greggio, L. 1999, AJ, 118, 302

Babul, A., Ferguson, H.C. 1996, ApJ, 458, 100

Babul, A., Rees, M. 1992, MNRAS, 255, 346

Bertelli, G., Bressan, A., Chiosi, C., Fagotto, F., Nasi, F. 1994 A\&AS, 106, 275

Bessell, M.S., Castelli, F., Plez, B. 1998, A\&A, 333, 231

Bruzual, A.G., Charlot, S. 2000, in preparation

Burstein, D., Heiles, C., 1984, ApJS, 54, 33

Calzetti, D., Meurer, G., Bohlin, R., Garnett, D., Kinney, A., Leitherer, C., StorchiBergmann, T. 1997, AJ, 114, 1834

Cardelli, J.A., CLayton, g.C., Mathis, J.S. 1989, ApJ, 345, 245

Campbell, A.W., Terlevich, R. 1984, MNRAS, 211, 15

Condon, J. J. 1992, ARA\&A, 30, 575

Condon, J.J., Cotton, W.D., Greisen, E.W., Yin, Q.F., Perley, R.A., Taylor, G.B., Broderick, J.J. 1998, AJ, 115, 16

Cram, L., Hopkins, A., Mobasher, B., Rowan-Robinson, M. 1998, ApJ, 507, 155

Crone, M.M., Schulte-Ladbeck, R.E., Hopp, U., Greggio, L. 2000, ApJ, 545, L31

Deharveng, J.-M., Albrecht, R., Barbieri, C., Blades, J.C., Boksenberg, A., Crane, P., Disney, M.J., Jakobsen, P., Kamperman, T.M., King, L.R., Macchetto, F., Mackay, C.D., Paresce, F., Weigelt, G., Baxter, D., Greenfield, P., Jedrzejewski, R., Nota, A., Sparks, W.B. 1994, A\&A, 288, 413

Doublier, V., Kunth, D., Courbin, F., Magain, P. 2000, A\&A, 353, 887

Drozdovsky, I.O., Tikhonov, N. 2000, A\&AS, 142, 347 
Drozdovsky, I.O., Schulte-Ladbeck, R.E., Hopp, U., Crone, M.M., Greggio, L. 2001a, ApJL, submitted

Drozdovsky, I.O., Schulte-Ladbeck, R.E., Hopp, U., Greggio, L., Crone, M.M. 2001b, AJ, in preparation

Ferguson, H.C., Babul, A. 1998, MNRAS, 296, 585

Fanelli, M.N., O’Connell, R.W., Thuan, T.X. 1988, ApJ, 334, 665

Fagotto, F., Bressan, A., Bertelli, G., Chiosi, C. 1994, A\&AS, 104, 365

Gerola, H., Seiden, P.E., Schulman, L.S. 1980, ApJ, 242, 517

Girardi, L., Bressan, A., Bertelli, G., Chiosi, C. 2000, A\&AS, 141, 371

Grebel, E., 2000, astro-ph/0008249

Greggio, L. 1986, A\&A, 160, 111

Greggio, L., Tosi, M., Clampin, M., De Marchi, G., Leitherer, C., Nota, A., Sirianni, M. 1998, ApJ, 504, 725

Gronwall, C., Koo, D.C. 1995, ApJ, 440, L1

Guzmán, R., Jangren, A., Koo, D.S., Bershady, M.A., Simard, L. 1998, ApJL, 495, 13

Henry, R.B.C., Edmunds, M.G., Köppen, J. 2000, ApJ, 451, 660

Hunter, D. A. 2001, in preparation

Hunter, D. A., Gallagher, J. S. 1986, PASP, 98, 5

Hurley-Keller, D., Matero, M., Nemec, J. 1998, AJ, 115, 1840

Izotov, Y.I., Thuan, T.X. 1999, ApJ, 511, 639

Izotov, Y.I., Thuan, T.X. Lipovetsky, V.A. 1997, ApJS, 108, 1

Kobulnicky, H.A., Skillman, E.D. 1996, ApJ, 471, 211 
Kunth, D., Maurogordato, S., Vigroux, L. 1988, A\&A, 204, 10

Kunth, D., Östlin, G. 2000, A\&AR, 10, 1

Kunth, D., Sargent, W.L.W. 1986, ApJ, 300, 496

Lee, M.G., Freedman, W.L., Madore, B.F., 1993, ApJ, 417, 553

Legrand, F. 2000, NewAR, 44, 345

Legrand, F., Kunth, D., Roy, J.-R., Mas-Hesse, J. M., Walsh, J.R. 2000, A\&A, 355, 891

Loose, H.H., Thuan, T.X. 1986, in Star-Forming Dwarf Galaxies and Related Objects, ed. D. Kunth, T.X. Thuan, and J. Tran Than Van (Gif-sur-Yvette: Éditions Frontières), 73

Lynds, R., Tolstoy, E., O’Neil Jr., E.J., Hunter, D.A. 1998, AJ, 116, 146

Makarova, L.N., Karachentsev, I.D., Georgiev, Ts.B. 1997, AstL, 23, 378

Marconi, G., Matteucci, F. Tosi, M. 1994, MNRAS 270, 35

Mas-Hesse, J.M., Kunth, D. 1999, A\&A, 349, 765

Östlin, G. 2000, ApJL, 535, 99

Origlia, L., Leitherer, C. 2000, AJ, 119, 2018

Origlia, L., Oliva, E. 1998, in "Dwarf Galaxies and Cosmology", ed. T.X. Thuan, C. Balkowski, V. Cayatte, and J. Tran Than Van (Gif-sur-Yvette: Éditions Frontières), 167

Papaderos, P., Loose, H.-H., Thuan, T.X., Fricke, K.J. 1996, A\&AS, 120, 207

Phillips, S., Driver, S. 1995, MNRAS, 274, 832

Ratnatunga, K.U., Bahcall, J.N. 1985, ApJS, 59, 63

Rocca-Volmerange, B., Guideroni, B. 1990, MNRAS, 247, 166

Sakai S., Madore B.F., \& Freedman W.L., 1996, ApJ, 461, 713 
Sargent, W.L.W., Searle, L. 1970, ApJL, 162, 155

Schlegel, D.J., Finkbeiner, D.P., Davis, M. 1998, ApJ, 500, 525

Schmidt, A.A., Alloin, D., Bica, E. 1995, MNRAS, 273, 945

Schulte-Ladbeck, R.E., Crone, M.M., Hopp, U. 1998, ApJ, 493, L23 (SCH98)

Schulte-Ladbeck, R.E., Hopp, U., Crone, M.M. Greggio, L. 1999, ApJ, 525, 709 (SHCG99)

Schulte-Ladbeck, R.E., Hopp, U., Greggio, L., Crone, M.M. 1999, AJ, 118, 2705 (SHGC99)

Schulte-Ladbeck, R.E., Hopp, U., Greggio, L., Crone, M.M. 2000, AJ, in press (SHGC00)

Searle, L., Sargent, W.L.W. 1972, ApJ, 173, 25

Searle, L., Sargent, W.L.W., Bagnuolo, W.G. 1973, ApJ, 179, 426

Stetson, P.B., Davis, L.E., Crabtree, D.B. 1990, in ASP Conf. Proc. 8, CCDs in Astronomy, ed. G.H. Jacoby (San Francisco:ASP), 289

Smecker-Hane, T.A., Stetson, P.B., Hesser, J.E., Lehnert, M.D. 1994, AJ, 108, 507

Sweigart A.V., Greggio, L., Renzini A. 1990, ApJ, 364, 527

Tacconi, L.J., Young, J.S. 1987, ApJ, 322, 681

Telles, E., Melnick, J., Terlevich, R. 1997, MNRAS, 288, 78

Thuan T.X. 1991, in "Massive Stars in Starbursts", ed. Leitherer C., Walborn N.R., Heckman T.M. and Norman C.A. (Cambridge Univ. Press: Cambridge), 183

Thuan T.X., Lipovetsky, V.A., Martin, J.-M., Pustil'nik, S.A. 1999, A\&AS, 139, 1

Tosi, M. 1998, in "Dwarf Galaxies and Cosmology", ed. T.X. Thuan, C. Balkowski, V. Cayatte, and J. Tran Than Van (Gif-sur-Yvette: Éditions Frontières), 443

Tosi, M., Greggio, L., Marconi, G., Focardi, P. 1991, AJ, 202, 951 
Trams, N.R., van Loon, J.Th., Waters, L.B.F.M., Zijlstra, A.A., Loup, C., Whitelock, P.A., Groenewegen, M.A.T., Blommaert, J.A.D.L., Siebenmorgen, R., Heske, A., Feast, M.W. 1999, A\&A, 346, 843

Viallefond, F., Thuan, T.X. 1983, ApJ, 269, 444

van Zee, L., Skillman, E.D., Salzer, J.J. 1998, AJ, 116, 1186 
Fig. 1. - The 103a-E image of I Zw 36 from the Digitized Sky Survey in grey-scale representation. The brightest feature is a blend of several H-II complexes. This is where the NIC2 (red) and FOC (blue) cameras were pointed. The faint, elliptical main body can also be seen. The NIC1 (small red box) was pointed in the background sheet.

Fig. 2.- a) NIC2 image composed by combining all images taken through the F110W filter. b) Same for images taken through the F160W filter. The galaxy is clearly resolved into stars.

Fig. 3.- The DAOPHOT-derived photometric errors.

Fig. 4.- Completeness fractions from ADDSTAR tests.

Fig. 5.- Color-magnitude diagrams of I Zw 36 in magnitudes in the HST Vegamag system. The location of the TRGB is indicated.

Fig. 6.- Color-magnitude diagrams of I Zw 36 after transformation to ground-based J and H. The foreground extinction is negligible.

Fig. 7.- Luminosity functions of I Zw 36 in instrumental HST magnitudes in the Vegamag system. The location of the adopted TRGB is marked.

Fig. 8.- Color-magnitude diagrams of I Zw 36 with three cuts applied on the size of the photometric errors $(1 \sigma)$ in both $\mathrm{J}$ and $\mathrm{H}$.

Fig. 9.- Color-magnitude diagrams of I Zw 36 with two sets of tracks overlayed. The first set of tracks is for a metallicity of $\mathrm{Z}=0.0004$ and the corresponding short distance modulus of 28.8. The second set is for $\mathrm{Z}=0.004$ and the long distance modulus of 29.5. For each case, the TRGB is indicated, and the corresponding absolute-magnitude scale is given on the right-hand ordinate.

Fig. 10. - NIC2 camera X- and Y-positions in the blue plume (plotted here are stars with 
$\left.(\mathrm{J}-\mathrm{H})_{0}<0.20\right)$ and in the red tangle $\left((\mathrm{J}-\mathrm{H})_{0}>0.5\right.$ and $\mathrm{H}_{0}$ larger than the TRGB). The young, massive stars in the blue plume occupy the central part of the image, in the same region where the FOC images also show massive stars and ionized gas (compare Figs. 1 and 2). The stars in the red tangle fill the image almost uniformly (with the exception of those central portions occupied by H-II regions where there is more crowding). This is the morphological feature that we refer to as Baade's red sheet.

Fig. 11. - The near-UV - optical, FOC CMD of I Zw 36 from Deharveng et al. (1994) is compared with the near-IR, NIC2 CMD. The CMDs are plotted in such a way that their blue and red plumes roughly overlay. Notice the blue plume dominates the near-UV - optical CMD, while the red plume is the most prominent feature of the near-IR CMD. This may result in color biasing of interpretations of CMDs.

Fig. 12. - A comparison of the near-IR CMDs of VII Zw 403, Mrk 178, and I Zw 36. For more details, refer to the text. The TRGB is indicated by a dashed green line. The dashed blue line indicates the location of the blue plume. The black dashed line is our dividing line between RSG (left) and AGB (right) stars.

Fig. 13. - Exemplary, simulated CMDs for I Zw 36, using our $\mathrm{Z}=0.0004$ simulator. Case (a) - continuous star formation from 2 Gyr ago until today. Cases (b)-(e), quiescent periods of $10,100,500$, and 1000 Myr prior to the present star formation. The CMD morphology of cases (d) and (e) clearly differs from that of the data, effectively ruling out any long discontinuities in the recent star formation history of I Zw 36.

Fig. 14. - We judge the quality of a model by how well it reproduces the CMD morphology and the luminosity function of the data. The LF of the data is shown by the heavy black line. Starcounts are given in 0.25 mag. intervals; the errorbars drawn are \pm the squareroot of the starcount in each bin. Several models are overplotted on the data. Notice how the model 
with a 2-Gyr-continuous SFR provides a good overall match to the data. We can interpret this galaxy as a "young" galaxy. Several lines of evidence indicate the interpretation that the galaxy is "ancient" is also possible. However, a model with a 15-Gyr-continuous SFR does not match the data; there is too much power in the old stellar component at the TRGB and too little power in the young and luminous stellar content. On the other hand, a good match is achieved when the powers are adjusted such that $\mathrm{SFR} /<\mathrm{SFR}>_{\text {past }}=13$. This is illustrated by the 14+1-Gyr-continuous SFR model. 
This figure "sl_zw_fig1.gif" is available in "gif" format from: http://arxiv.org/ps/astro-ph/0102397v1 
This figure "sl_zw_fig2a.gif" is available in "gif" format from: http://arxiv.org/ps/astro-ph/0102397v1 
This figure "sl_zw_fig2b.gif" is available in "gif" format from: http://arxiv.org/ps/astro-ph/0102397v1 


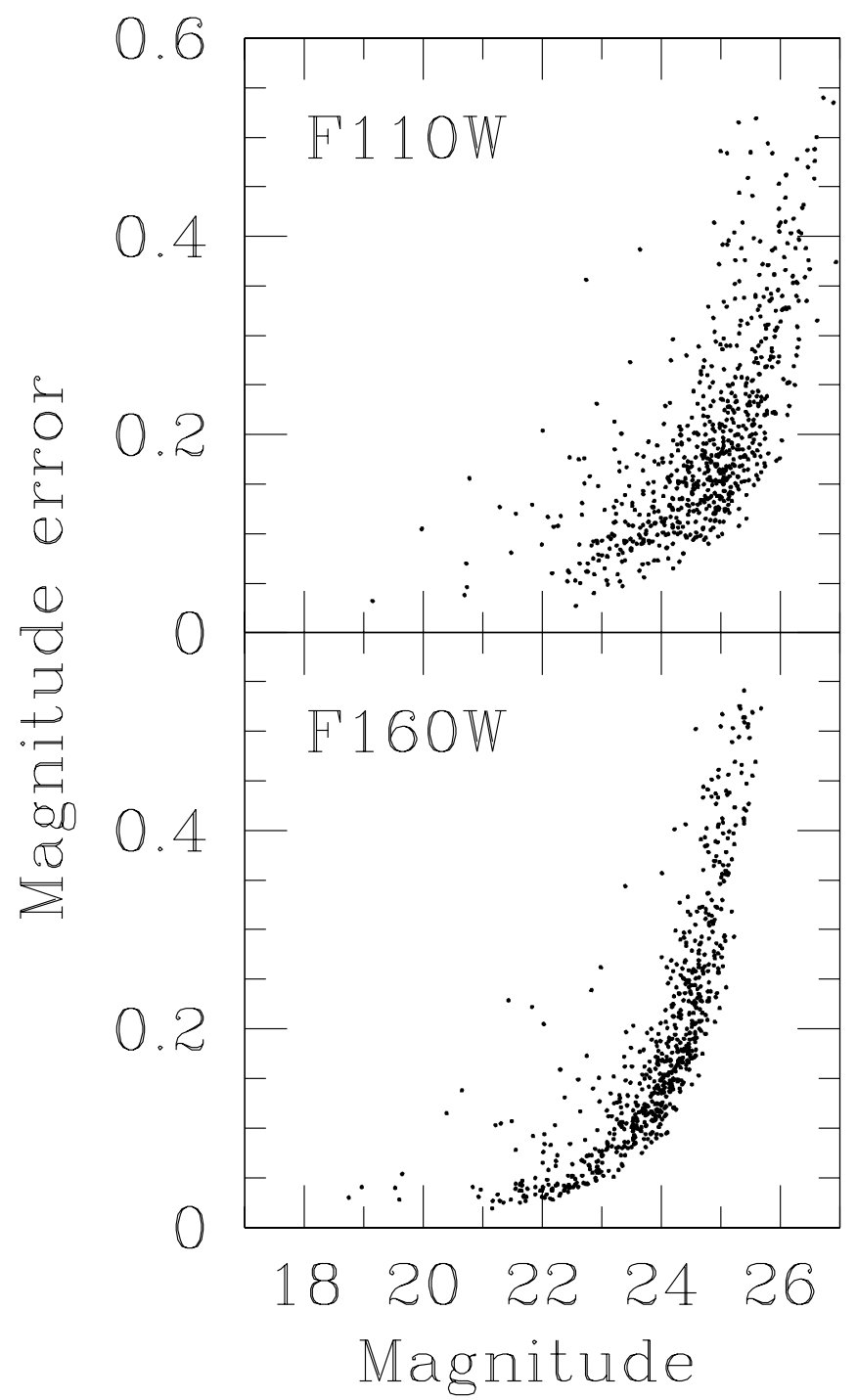




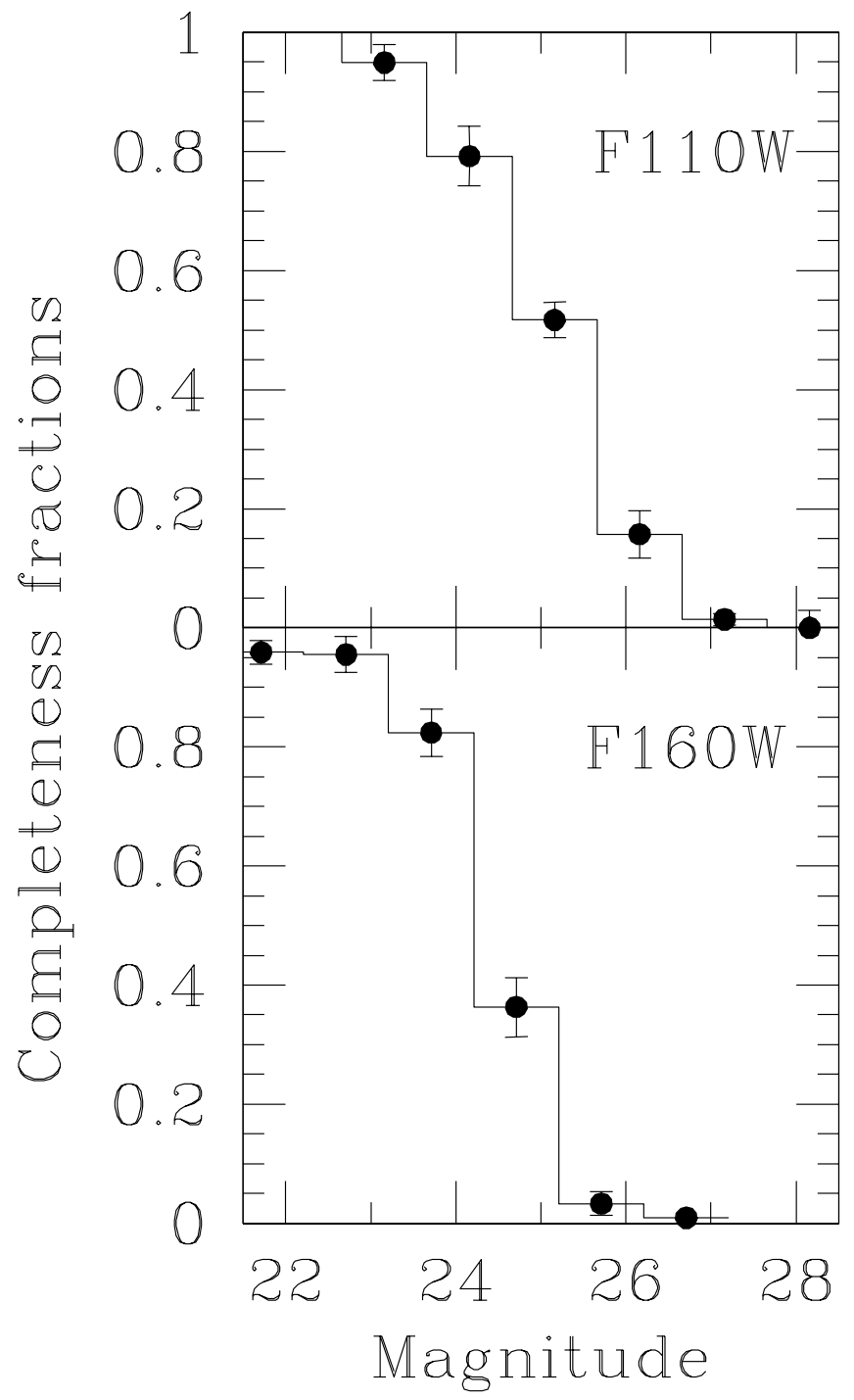



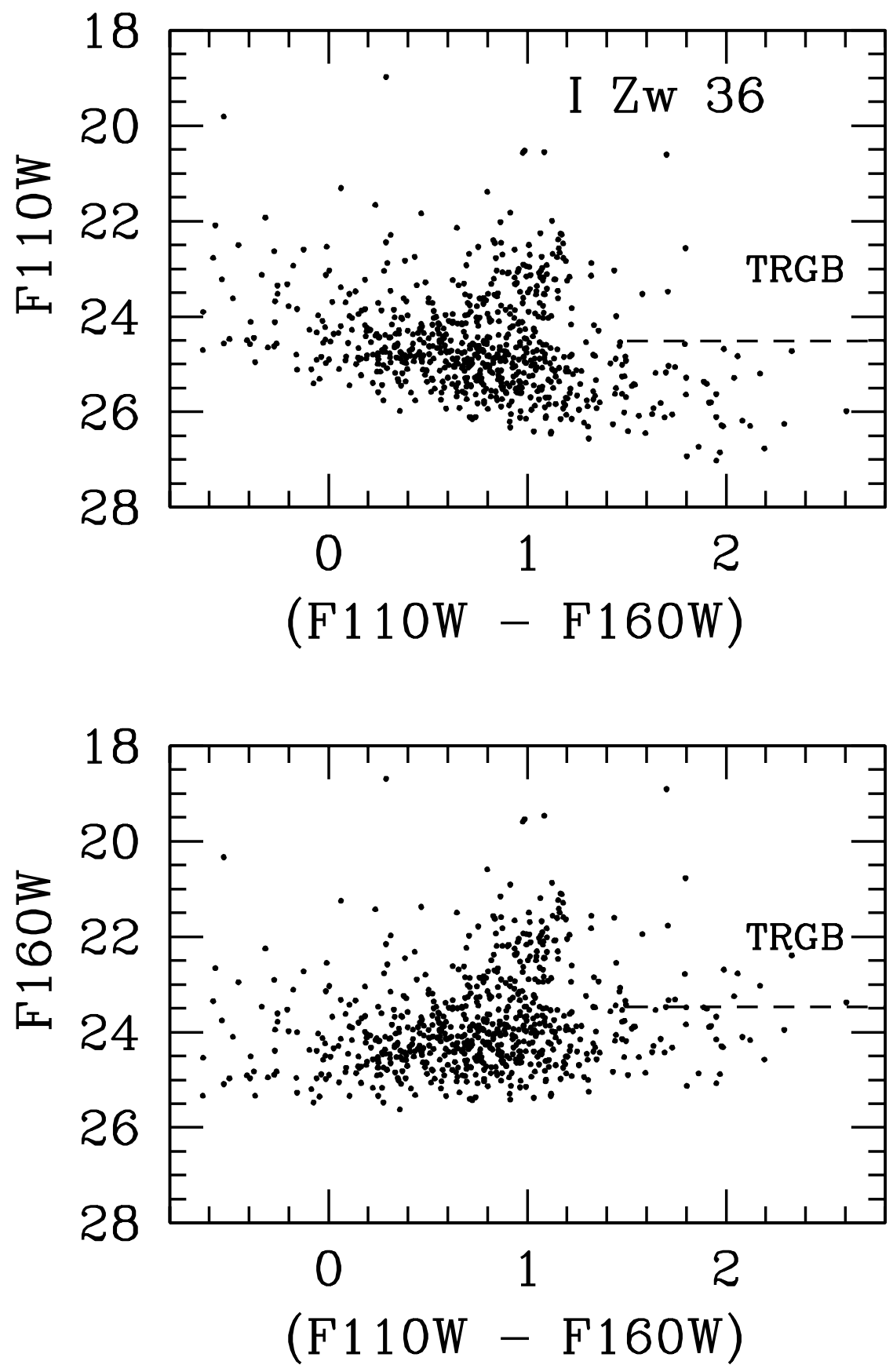

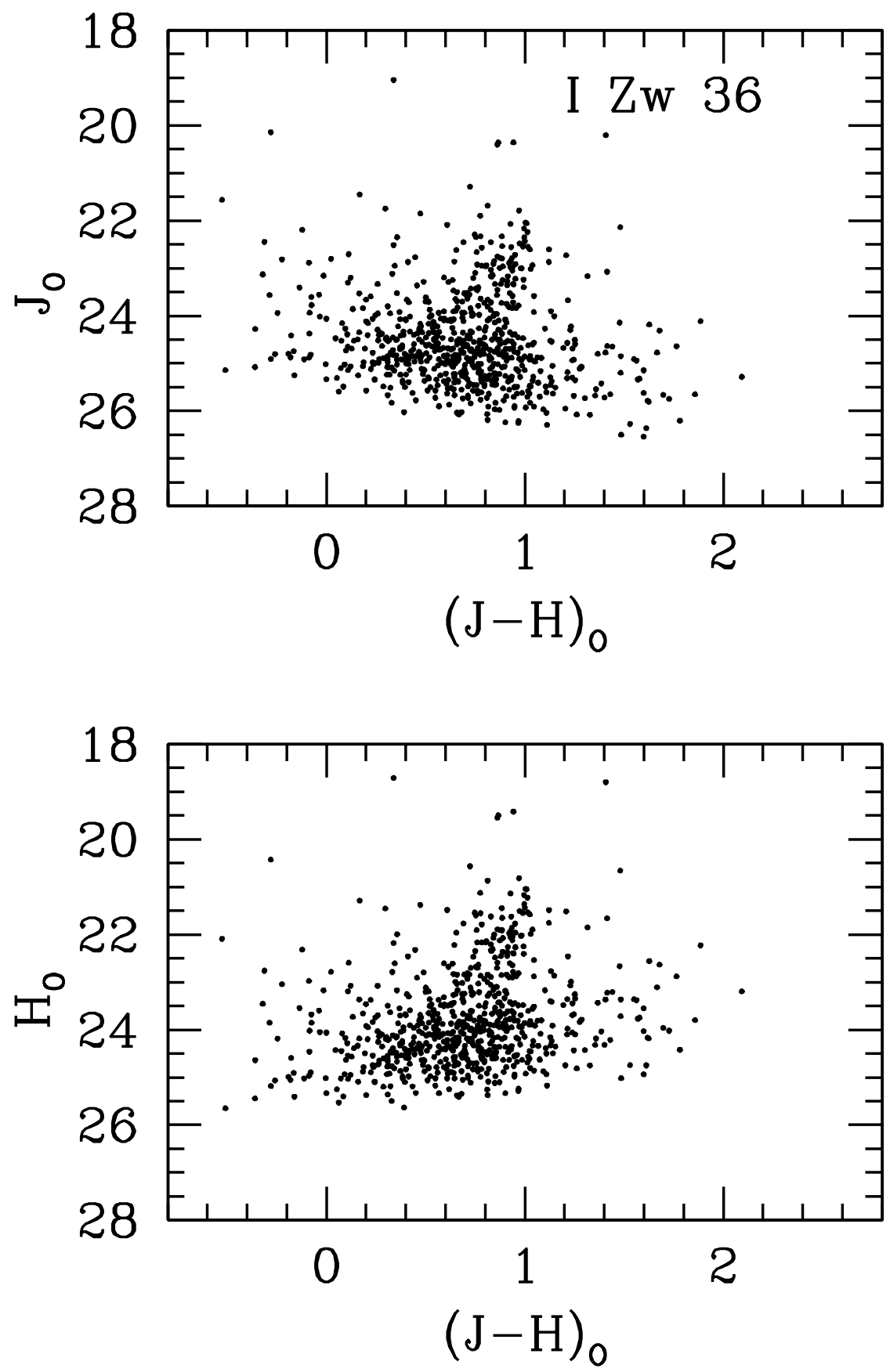


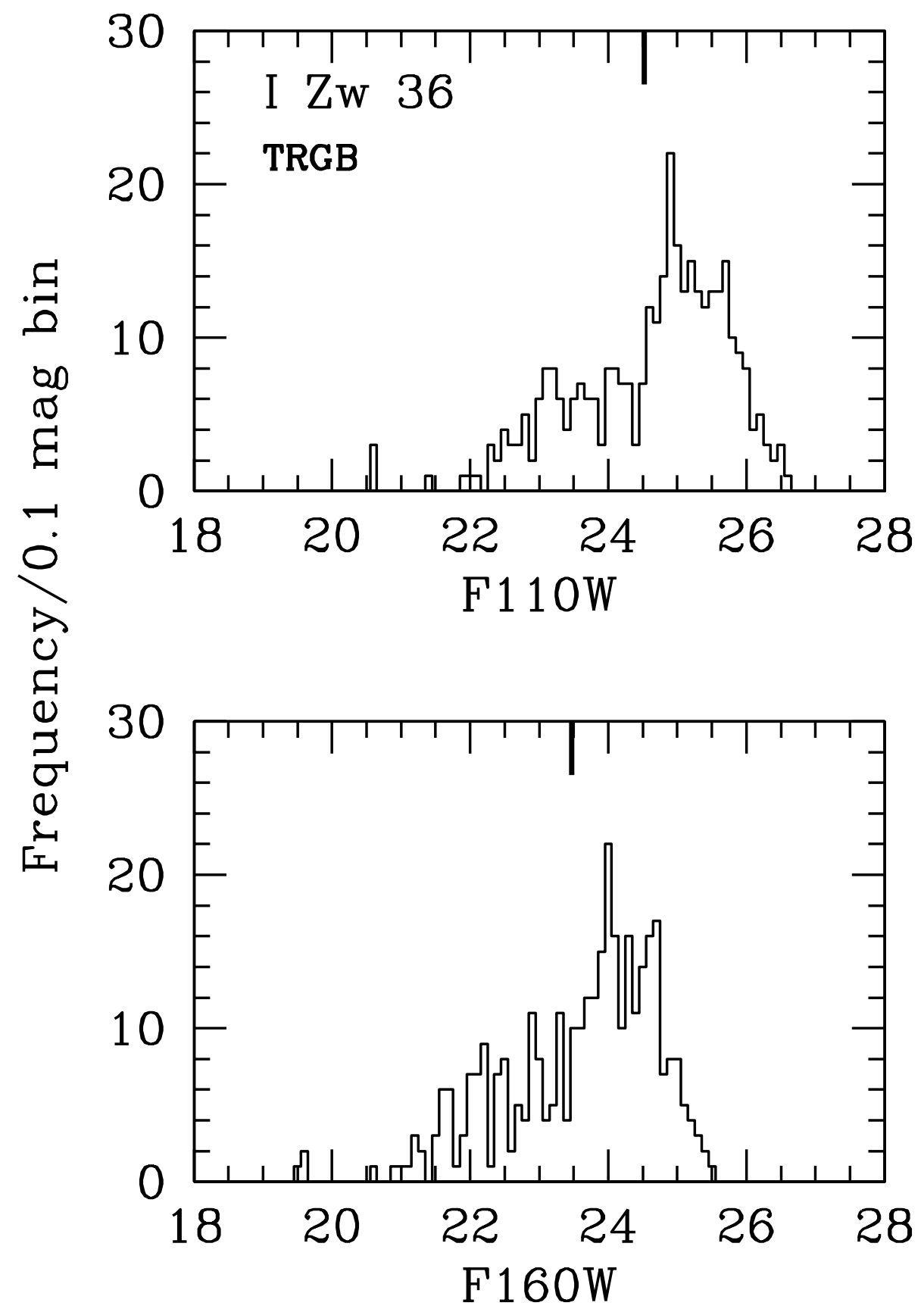




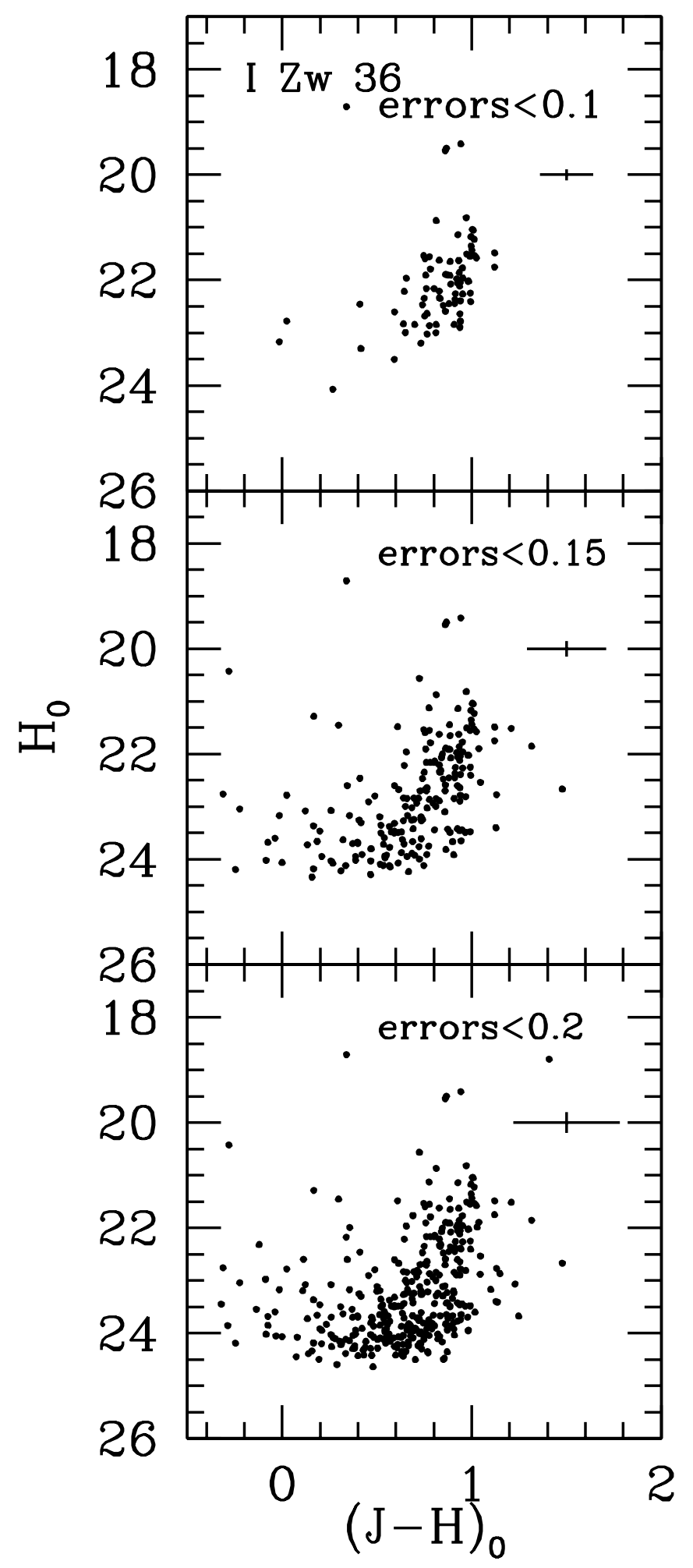



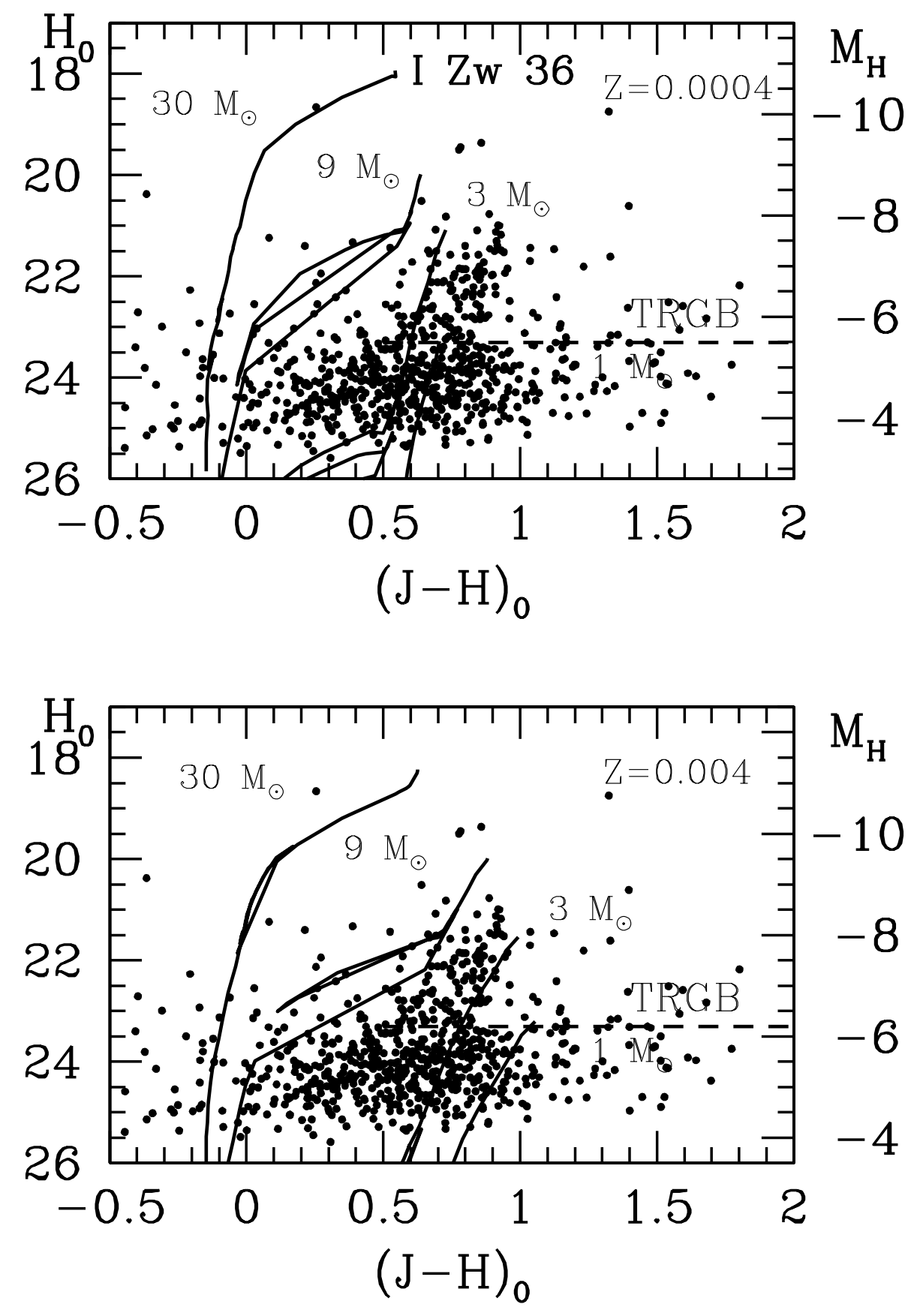


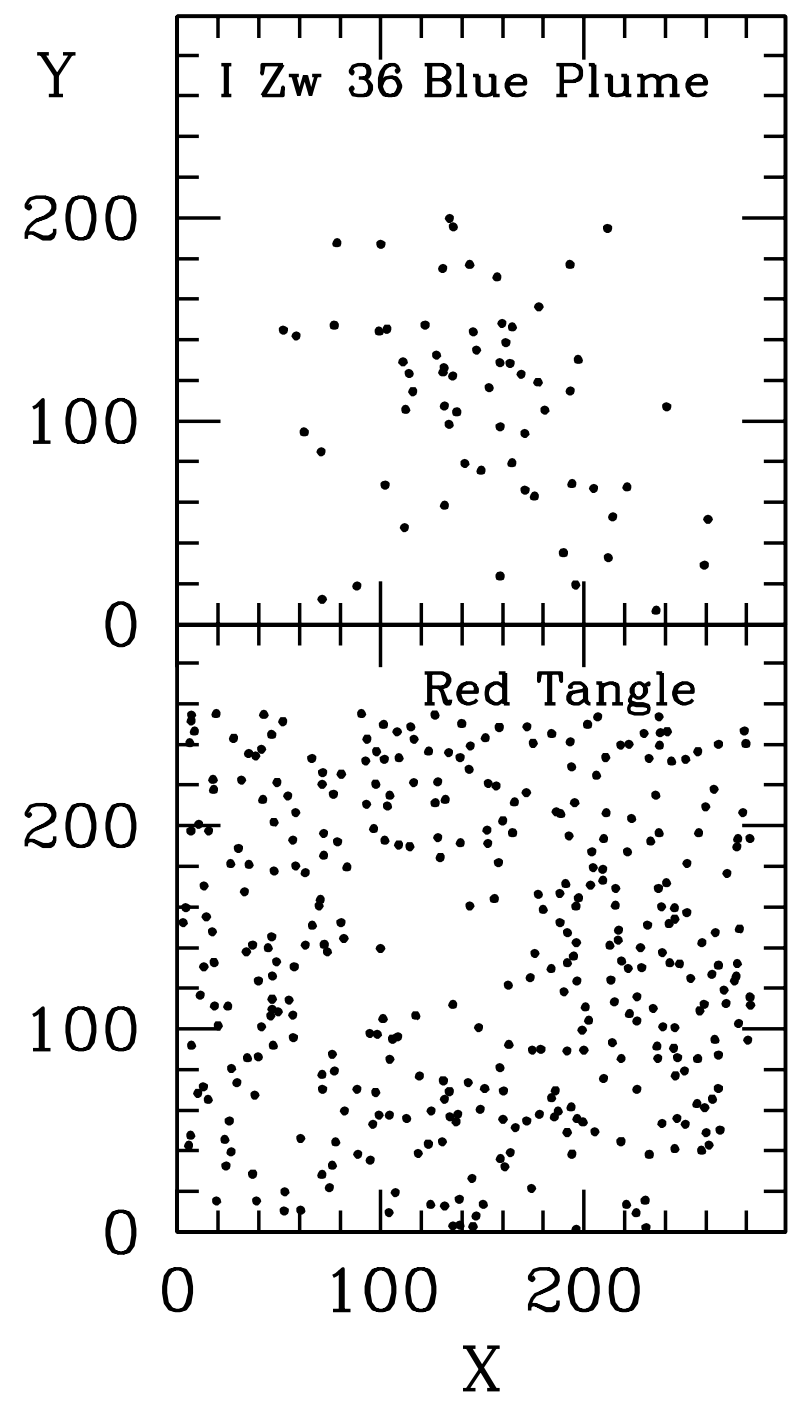



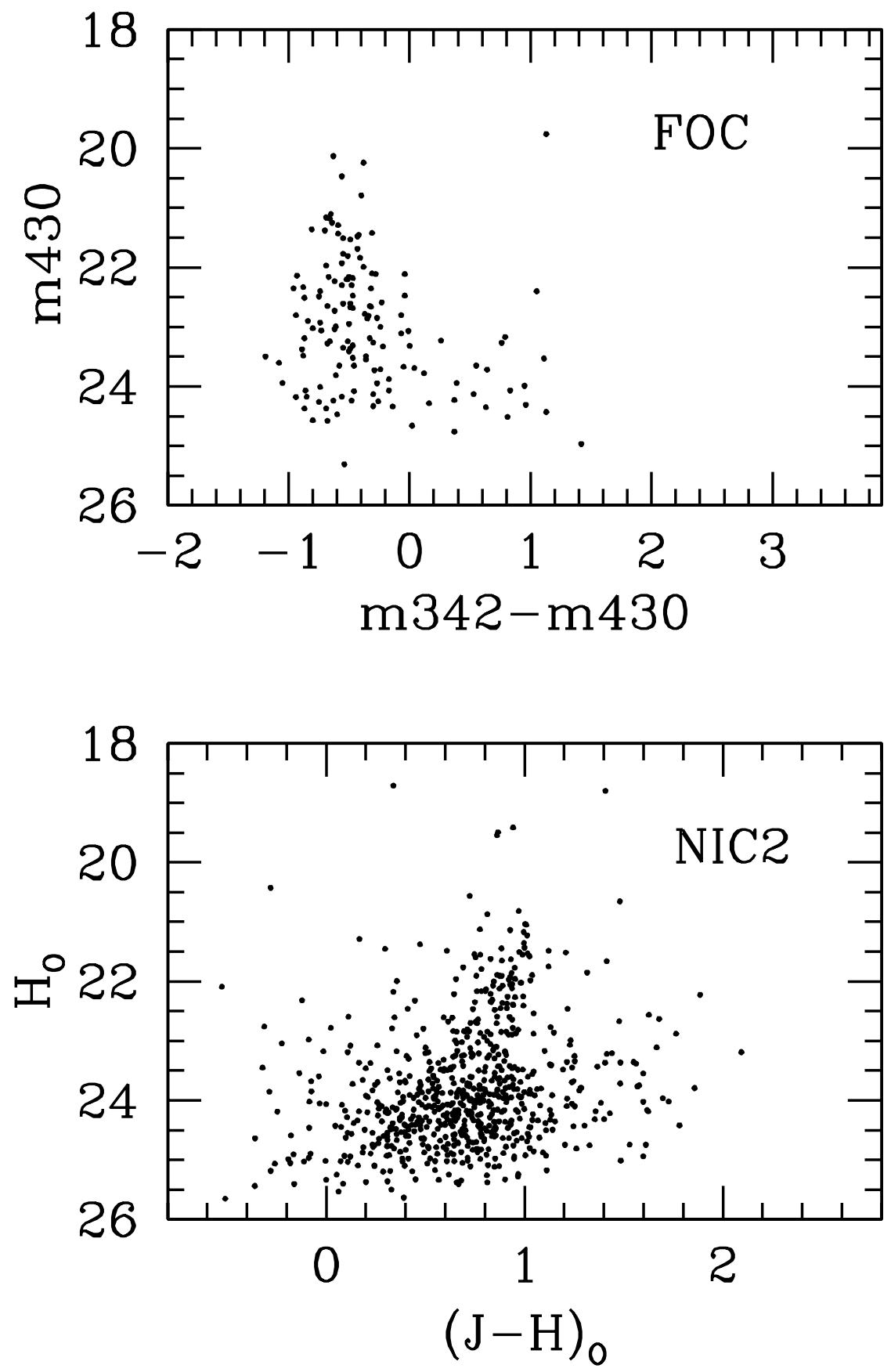
This figure "sl_zw_fig12.gif" is available in "gif" format from: http://arxiv.org/ps/astro-ph/0102397v1 
This figure "sl_zw_fig13.gif" is available in "gif" format from: http://arxiv.org/ps/astro-ph/0102397v1 


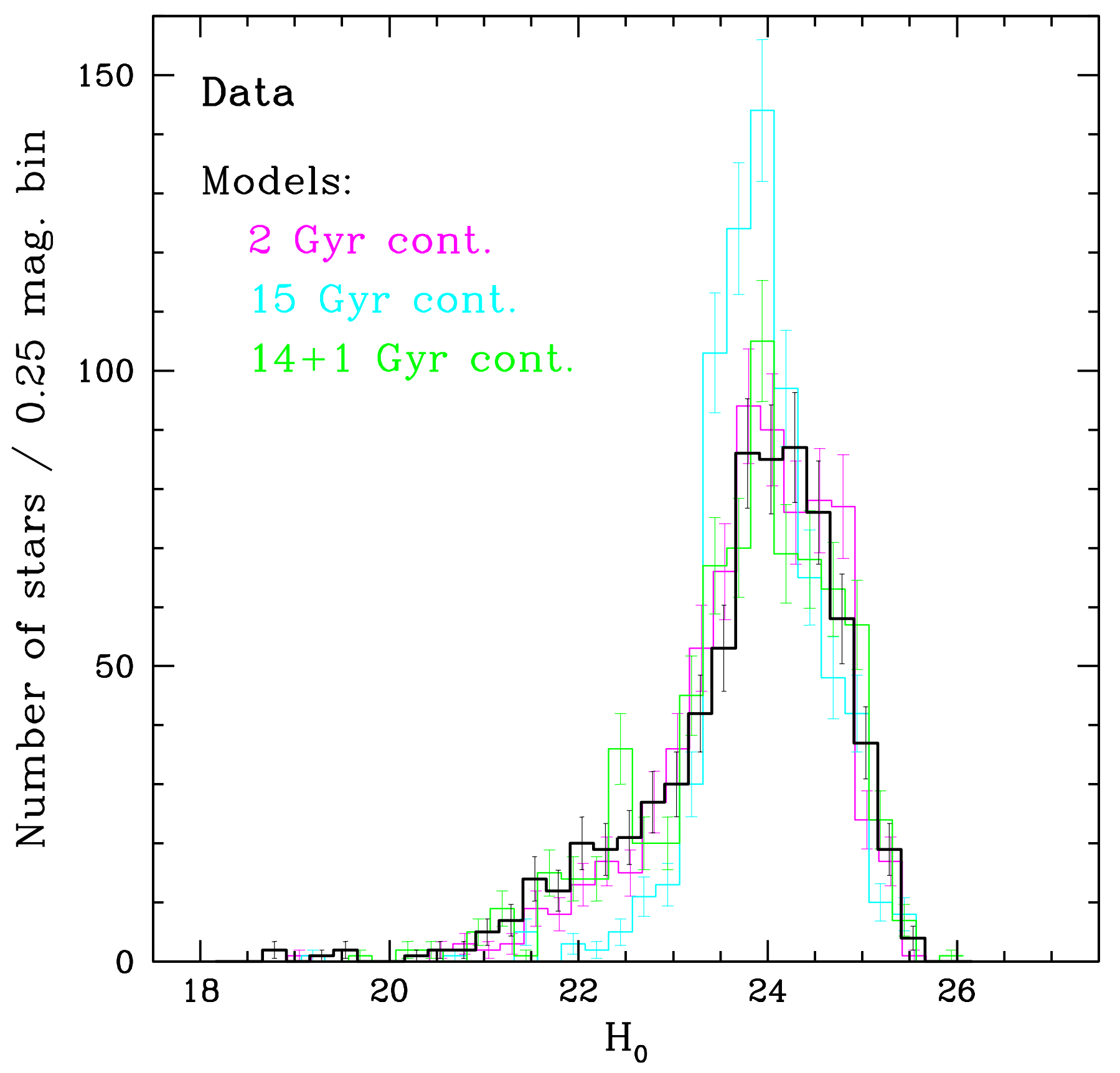

